Métodos híbridos para o problema de dimensionamento de lotes com múltiplas plantas 

SERVIÇO DE PÓS-GRADUAÇÃO DO ICMC-USP

Data de Depósito:

Assinatura:

\title{
Métodos híbridos para o problema de dimensionamento de lotes com múltiplas plantas ${ }^{(1)}$
}

\author{
Daniel Henrique Silva \\ Orientadora: Franklina Maria Bragion de Toledo
}

Dissertação apresentada ao Instituto de Ciências Matemáticas e de Computação - ICMC-USP, como parte dos requisitos para obtenção do título de Mestre em Ciências - Ciências da Computação e Matemática Computacional. VERSÃO REVISADA

USP - São Carlos

Março de 2013

${ }^{(1)}$ Este trabalho contou com o apoio financeiro do CNPq e da FAPESP 
Ficha catalográfica elaborada pela Biblioteca Prof. Achille Bassi e Seção Técnica de Informática, ICMC/USP, com os dados fornecidos pelo(a) autor(a)

Silva, Daniel

SS586M Métodos híbridos para o problema de dimensionamento de lotes com múltiplas plantas / Daniel Silva; orientadora Franklina Toledo. -- São Carlos, 2013. $53 \mathrm{p}$.

Dissertação (Mestrado - Programa de Pós-Graduação en Ciências de Computação e Matemática Computacional) Instituto de ciências Matemáticas e de computação, Universidade de São Paulo, 2013.

1. Otimização inteira. 2. Métodos heurísticos. I. Toledo, Franklina, orient. II. Título. 
À minha família 
Good name in man and woman, dear my lord, Is the immediate jewel of their souls: Who steals my purse steals trash; 'tis something, nothing;

'Twas mine, 'tis his, and has been slave to thousands; But he that filches from me my good name, Robs me of that which not enriches him, And makes me poor indeed. W. Shakespeare 


\section{Agradecimentos}

Agradeço primeiramente, à minha família, Tânia, Iracema e Danilo, cujo apoio e amor foram essenciais, não somente durante os dois últimos anos, mas por toda a minha vida,

À professora Franklina, que acreditou em mim, até nos momentos em que eu próprio havia deixado de acreditar,

À minha namorada Sarita, e cada momento maravilhoso que eu tive chance de passar ao seu lado, e cada conversa inspiradora que tivermos,

Aos demais professores do LOT, com os quais eu aprendi muito,

Ao pessoal do laboratório de otimização, pelo trabalho no último ano, em especial ao Luiz Henrique Cherri, cuja ajuda foi essencial para a conclusão deste projeto,

Aos amigos de longa data, que compartilharam momentos bons e ruins, e compreenderam a minha ausência por estes últimos dois anos, em especial, ao pessoal do grupo de Go,

Aos novos amigos, que apoiaram essa jornada,

A todos os professores que me inspiraram no passado, e me ajudaram a me tornar o que eu sou hoje,

A Pitoquinha, que sempre me recebeu feliz a cada retorno para casa,

Finalmente, à Deus, por colocar todos eles em meu caminho.

E ao CNPq e à FAPESP, pelo apoio financeiro. 


\section{Resumo}

Neste trabalho, apresentamos um estudo sobre o problema de dimensionamento de lotes com múltiplas plantas, múltiplos itens e múltiplos períodos. As plantas têm capacidade de produção limitada e a fabricação de cada produto incorre em tempo e custo de preparação de máquina. Nosso objetivo é encontrar um plano de produção que satisfaça a demanda de todos os clientes, considerando que a soma dos custos de produção, de estoque, de transporte e de preparação de máquina seja a menor possível. Este trabalho tem duas contribuições centrais. Primeiramente, propomos a modelagem do problema de dimensionamento de lotes com múltiplas plantas utilizando o conceito de localização de facilidades. Para instâncias de pequena dimensão, os testes computacionais mostraram que a resolução do problema remodelado apresenta, como esperado, resultados melhores que o modelo original. No entanto, seu elevado número de restrições e de variáveis faz com que as instâncias de maiores magnitudes não consigam ser resolvidas. Para trabalhar com instâncias maiores, propomos um método híbrido (math-heurística), que combina o método relax-and-fix, com a restrição de local branching. Testes computacionais mostram que o método proposto apresenta soluções factíveis de boa qualidade para estas instâncias.

Palavras-chaves: dimensionamento de lotes, múltiplas plantas, otimização inteira-mista, math-heurística. 


\section{Abstract}

In this work, we present a study about the multi-plant, multi-item, multi-period lot-sizing problem. The plants have limited capacity, and the production of each item implies in setup times and setup costs. Our objective is to find a production plan which satisfies the demand of every client, considering that the sum of the production, stocking, transport and setup costs is the lowest possible. This work has two main contributions. Firstly, we propose the multi-plant lot-sizing problem modeling using the facility location concept. For small dimension problems, computational tests showed that the remodeled problem resolution presents, as expected, better results than the original model. However, the great number of restrictions and variables make bigger instances to be intractable. To work with the bigger dimension instances, we propose a hybrid method (math-heuristic), which combines the relax-and-fix method and the local branching restriction. Computational tests show that the proposed math-heuristic presents good quality feasible solutions for these instances

Keywords: Lot-sizing, multi-plant, mixed-integer optimization, math-heuristic. 


\section{Sumário}

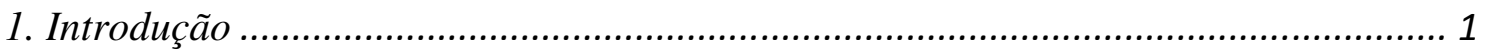

2. O Problema de Dimensionamento de Lotes com Múltiplas Plantas ............................ 3

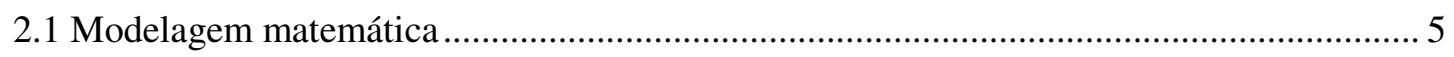

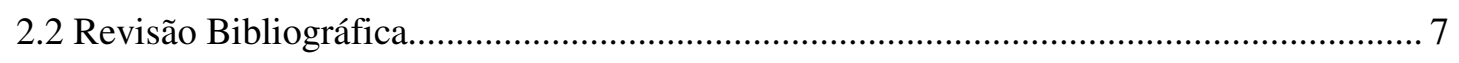

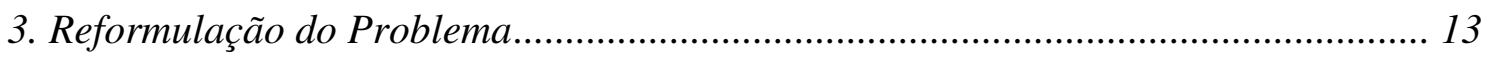

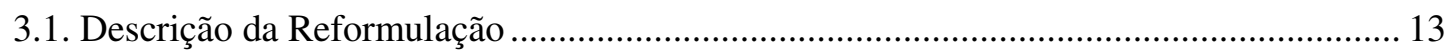

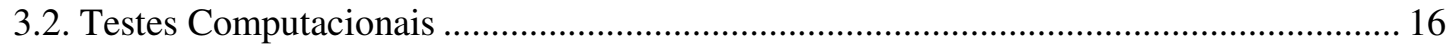

3.2.1. Análise da Relaxação Linear dos Modelos .......................................................................... 18

3.2.2. Análise do Desempenho do Programa Inteiro Misto ............................................................... 25

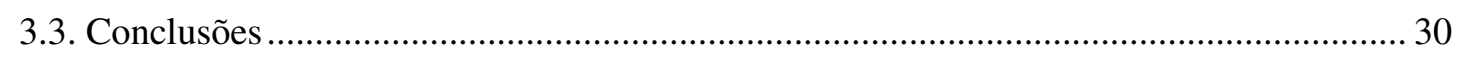

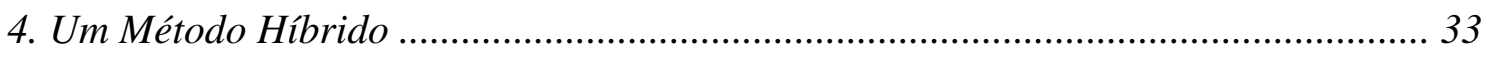

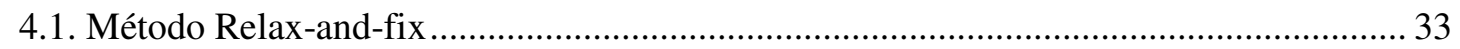

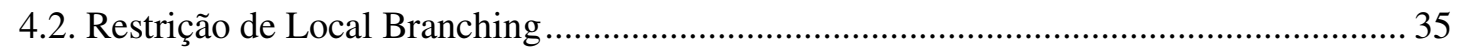

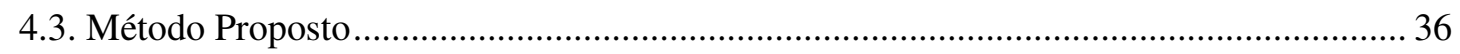

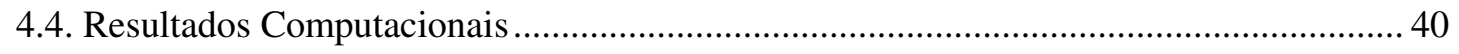

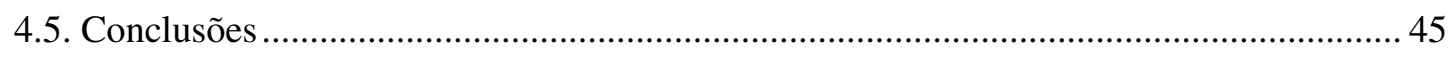

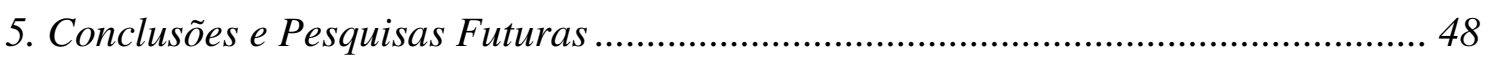

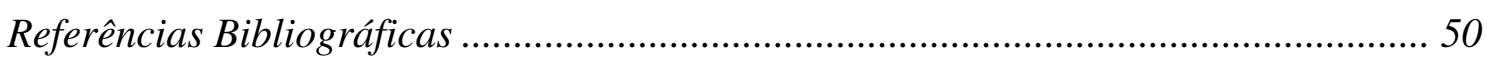




\section{Introdução}

Nos tempos atuais, se faz cada vez mais necessária a melhor utilização dos recursos de produção, não somente pelo fator econômico, mas também pela crescente preocupação com o meio ambiente. Deste modo, um eficiente planejamento da produção pode contribuir significativamente para o desenvolvimento sustentável.

O planejamento de produção envolve a aplicação de estratégias que controlem a produção em todos os seus estágios, desde a compra de matérias-primas, até a quantidade de cada produto fabricado, bem como a sua distribuição aos consumidores finais, incluindo as etapas intermediárias de produção, coordenação dos turnos de trabalho, quantidade de mão-de-obra necessária para a produção, e manutenção do maquinário. O objetivo de traçar um plano de produção é, em geral, minimizar os recursos envolvidos no processo de produção.

O problema de dimensionamento de lotes consiste basicamente em, dada uma linha de produção, com capacidade de produção e demanda conhecidas, obter um planejamento de produção que determine quais produtos devem ser produzidos, em que ordem, e em que quantidade, dentro de um horizonte de produção pré-determinado. $\mathrm{O}$ objetivo é determinar um plano de produção que satisfaça todas as características determinadas, com o menor custo possível.

É possível subdividir os problemas de dimensionamento de lotes em classes, de acordo com suas características específicas. Os problemas podem tratar da produção de um único produto, ou de múltiplos produtos, produtos estes que podem ser independentes ou dependentes entre si. Pode haver uma única linha de produção, ou podem haver linhas de produção paralelas, ou ainda linhas de produção dependentes entre si. Boas reviões sobre o assunto são encontradas em (Drexl \& Kimms, 1997), (Karimi, et al., 2003), (Brahimi, et al., 2006), (Jans \& Degraeve, 2008) e (Buschkühl, et al., 2010).

Algumas empresas de grande porte possuem mais de uma planta que podem estar posicionadas em diferentes locais (cidades, estados e até países). A fim de atender a demanda dos clientes de cada planta, os produtos produzidos por estas diferentes plantas podem ser transportados entre elas, ou seja, se for viável economicamente pode haver troca de produtos entre plantas.

Para uma empresa com múltiplas plantas, um planejamento de produção coordenado, considerando simultaneamente a produção, o estoque e o transporte trará 
melhores resultados do que o planejamento individual de cada planta, uma vez que considerando-se as necessidades globalmente, os resultados serão no mínimo tão bons quanto o tratamento individual de cada planta separadamente. No entanto, coordenar o planejamento de produção integrado é uma tarefa muito complexa, pelas próprias dimensões do problema.

O objetivo deste trabalho é propor um modelo eficiente para determinar uma solução ótima para o problema em tempo razoável. Caso isto não seja viável no tempo disponível, apresentamos um método heurístico para obter soluções de boa qualidade.

O restante desta dissertação se encontra dividido da seguinte maneira: No Capítulo 2, descrevemos detalhadamente o problema abordado, e fornecemos um primeiro modelo matemático para o problema, além de uma revisão bibliográfica, que fornece o estado da arte do assunto estudado. No Capítulo 3, propomos uma remodelagem para o problema e mostramos, através de testes computacionais, que esta remodelagem consegue resolver na otimalidade problemas de menores dimensões com baixos tempos computacionais, além de apresentar relaxações lineares de qualidade superior ao modelo apresentado no Capítulo 2. No Capítulo 4, propomos um método híbrido (math-heurística) baseada em relax-and-fix e local branching para a resolução de problemas de maiores dimensões. Testes computacionais que mostram que o método proposto determina resultados de boa qualidade são reportados. Finalmente, no Capítulo 5, apresentamos uma conclusão sobre o trabalho. 


\section{O Problema de Dimensionamento de Lotes com Múltiplas Plantas}

O dimensionamento de lotes é um problema de otimização combinatória com muitas aplicações práticas como, por exemplo, no planejamento de produção de peças em fundições (Camargo, et al., 2012), (Araujo, et al., 2008), (Santos-Meza, et al., 2002); na indústria de bebidas (Ferreira, et al., 2009); em fábricas para produção de ração animal (Toso, et al., 2009); na indústria de papel (Poltroniere, et al., 2008) e na distribuição de água (Toledo, et al., 2008).

O problema consiste basicamente em dada uma linha de produção com capacidade limitada e demandas conhecidas, obter um planejamento de quais produtos devem ser produzidos, em que quantidade em cada um dos períodos de um horizonte de planejamento finito. O objetivo do problema estudado é determinar um plano de produção de mínimo custo que atenda as demandas e respeite a capacidade da linha.

Em muitos problemas de dimensionamento de lotes, também é necessário considerar a preparação de máquina (setup), onde se considera o custo e o tempo necessários para a preparação da máquina para produção, quando esta deixa de produzir um produto e passa a produzir outro. O setup pode incluir a troca de matérias-primas dentro da máquina, o tempo de limpeza necessário em uma troca, a reorganização da produção, ou até mesmo a reprogramação da máquina. Trigeiro (1987) destaca a importância de considerar, além dos custos de preparação, os tempos gastos na preparação para produção.

Neste projeto foi estudada uma variante do problema de dimensionamento de lotes com múltiplas plantas. Tratamos do problema em que uma empresa possui diferentes plantas, em diferentes locais, cada qual capaz de produzir diferentes produtos. O transporte de produtos entre as plantas é permitido, no entanto, está associado um custo. Cada planta possui uma demanda própria para cada produto, em cada período de um horizonte de produção finito. A empresa pode produzir os produtos em qualquer uma das plantas. No entanto, os custos de transporte destes produtos entre as plantas são acrescentados ao custo total de produção, que deve ser minimizado. Portanto, o problema envolve a minimização dos custos de produção, de preparação, de estocagem e de transporte de produção. A Figura 2.1 ilustra um plano de produção para um problema com dois períodos, três plantas e três produtos. 


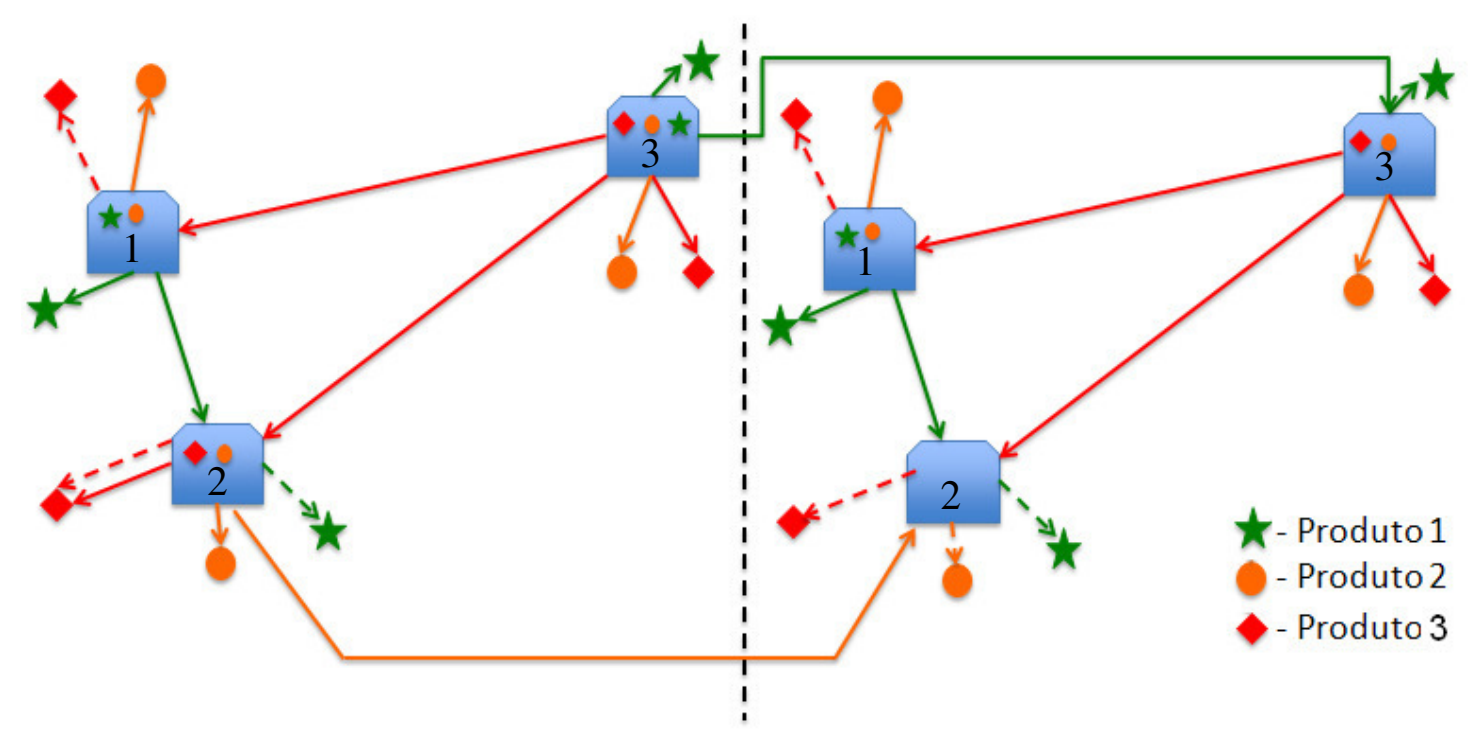

Primeiro Período

Segundo Período

Figura 2.1 - Plano de produção - exemplo.

Na Figura 2.1, a linha pontilhada vertical separa os dois períodos de produção. Cada retângulo azul representa uma planta, os símbolos (losango, círculo, estrela) ilustram os produtos. A produção de um produto numa dada planta em cada período é descrita pelo desenho deste produto no interior da planta. Linhas cheias entre as plantas indicam transporte de produtos entre elas. Linhas cheias entre períodos indicam estoque de produtos entre períodos. Linhas cheias saindo da planta indicam atendimento de demanda do produto por produção própria, enquanto as linhas pontilhadas indicam o atendimento da demanda de um produto pela produção em outra planta (transporte). Note que a demanda de um produto pode ser atendida pela combinação de produção própria e produção em outra planta (ver planta 2, período 1).

Esse problema foi pouco estudado na literatura. Na Seção 2.1, é detalhado o modelo proposto por Sambasivan \& Yahya (2005) para o problema. Uma breve revisão bibliográfica sobre o problema de dimensionamento de lotes é apresentada na Seção 2.2. Nesta seção, é realizada uma revisão detalhada dos problemas com máquinas paralelas e com plantas paralelas, sendo descritas brevemente outras configurações mais simples do problema. 


\subsection{Modelagem matemática}

Como proposto em (Sambasivan \& Yahya, 2005), este problema pode ser modelado como:

\section{Dimensões do problema:}

$n$ : Número de produtos.

$m$ : Número de plantas.

T: Número de períodos no horizonte de planejamento.

\section{Índices:}

$i$ : Índice relativo aos produtos. $i \in I$;

$j, k$ : Índice relativo às plantas. $j, k \in J$;

$t$ : Índice relativo aos períodos. $t \in S$;

\section{Conjuntos:}

$I$ : Conjunto de índices inteiros. $\mathrm{I}=\{i \in \mathbb{Z}\{i \leq n\}=\{1,2, \ldots, n\}$;

$J$ : Conjunto de índices inteiros. $\mathrm{J}=[j \in \mathbb{Z} \mid j \leq m\}=\{1,2, \ldots, m\}$;

$S$ : Conjunto de índices inteiros. $S=\{t \in \mathbb{Z} \mid t \leq T\}=\{1,2, \ldots, T\}$;

\section{Parâmetros:}

$d_{i j t}$ : Demanda do produto $i$ na planta $j$ no período $t$;

$\operatorname{cap}_{j t}$ : Capacidade de produção da planta $j$ no período $t$ (em unidades de tempo);

$b_{i j}$ : Tempo necessário para produzir uma unidade do produto $i$ na planta $j$;

$f_{i j}$ : Tempo de preparação para produção do produto $i$ na planta $j$;

$c_{i j}$ : Custo de produção do produto $i$ na planta $j$;

$s_{i j}$ : Custo de preparação para a produção do produto $i$ na planta $j$;

$r_{j k}$ : Custo unitário de transporte de qualquer produto da planta $j$ para a planta $k$;

$h_{i j}$ : Custo unitário de estoque do produto $i$ na planta $j$;

$l i m_{i j t}$ : limite de produção do produto $i$ na planta $j$ no período $t$, dado por

$$
\lim _{\mathrm{ijt}}=\operatorname{Min}\left\{\frac{\operatorname{cap}_{j t}-f_{i j}}{b_{j t}} ; \sum_{j \in J} \sum_{r=t}^{T} d_{i j r}\right\}
$$




\section{Variáveis de decisão:}

$X_{i j t}$ : Quantidade do produto $i$ produzida na planta $j$ no período $t$;

$I_{i j t}$ : Quantidade do produto $i$ estocada na planta $j$ ao final do período $t$;

$W_{i j k t}$ : Quantidade do produto $i$ transportada da planta $j$ para a planta $k$ durante o período $t$

$Z_{i j t}$ : Variável binária que tem valor 1 quando há produção do produto $i$ na planta $j$ durante o período $t$, e é igual a zero, caso contrário.

Minimizar:

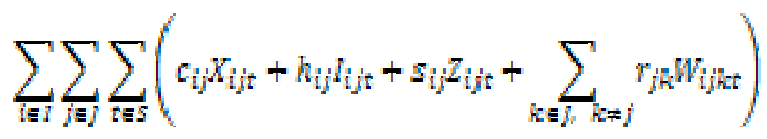

Sujeito a:

$$
\begin{array}{ll}
I_{i j t}=I_{i j(\mathrm{t}-1)}+X_{i j t}-\sum_{k \neq j} W_{i j k t}+\sum_{i \neq j} W_{i j t}-d_{i} \forall i \in I, \forall j \in J, \forall t \in S ;(2) \\
X_{i j t} \leq l i m_{i j t} Z_{i j t} & \forall i \in I, \forall j \in J, \forall t \in S ;(3) \\
\sum_{i \in I}\left(b_{i j} X_{i j t}+f_{i j} Z_{i j t}\right) \leq c a p_{j t} & \forall i \in I, \forall j \in J, \forall t \in S ;(4) \\
X_{i j t^{t}} I_{i j t} \geq 0 & \forall i \in I, \forall j \in J, \forall t \in S ;(5) \\
Z_{i j t} \in\{0 ; 1\} & \forall i \in I, \forall j \in J, \forall t \in S ;(6) \\
W_{i j k t} \geq 0 & \forall i \in I, \forall j, k \in J, \forall t \in(7)
\end{array}
$$

A função objetivo (1) visa minimizar a soma dos custos de produção, de estoque, de preparação e de transporte entre plantas, para todos os períodos do horizonte de produção. O grupo de restrições dado pelas equações (2) garante que as demandas serão atendidas sem atraso. $\mathrm{O}$ grupo de restrições dado pelo conjunto de inequações (3) impõe que a planta esteja preparada para a produção, toda vez que houver produção do produto a ela associada. O grupo de restrições dado pelo conjunto de inequações (4) garante que a capacidade de produção é respeitada. Finalmente, as restrições (5) (7) definem o domínio das variáveis. Vale notar que como as variáveis $I_{i j t}$ são todas não-negativas, então a demanda sempre será atendida no prazo, e não haverá nenhum atraso, ou seja, não há backlogging. 
Além disso, é importante ressaltar o fato de que neste modelo assume-se que toda a demanda transportada em um período de produção é entregue no próprio período de produção. Caso esta hipótese não seja admissível, podemos alterar as equações (2) de forma a considerar maiores tempos de entrega, isto é, supondo que o tempo de entrega entre a planta $j$ e a planta $k$ é dado por $t e_{k t}$ tem-se:

$$
I_{i j t}=I_{i j,(t-1)}+X_{i j t}-\sum_{k \neq j} W_{i j k t}+\sum_{l \neq j} W_{i l j\left(t-t e_{k t}\right)}-d_{i j t}
$$

Os problemas de dimensionamento de lotes com uma única planta, capacidade limitada, múltiplos produtos e tempos de preparação de máquina pertencem à classe de problemas NP - completos (Maes, et al., 1991). A partir desta prova, pode-se chegar a um resultado semelhante sobre a natureza do problema com múltiplas plantas.

Proposição: O problema de dimensionamento de lotes, com capacidade limitada, múltiplas plantas e tempo de preparação não-nulo é um problema que pertence à classe NP - completo.

Demonstração: Sabe-se que o problema de dimensionamento de lotes com uma única planta, capacidade limitada, tempo de preparação não-nulo pertence à classe NP completo (Maes, et al., 1991). Suponha para o modelo (1) (7) que as variáveis de transferência de produção entre as plantas sejam todas nulas (ou seja, assuma que $W_{i j k t}=$ $\left.0, \forall i, j, k_{r} t\right)$, este problema é claramente mais simples que o problema original, permitindo decompô-lo em $m$ problemas de dimensionamento de lotes com uma única planta, independentes entre si, cada qual com diversos produtos, tempo de preparação não-nulo e capacidade finita, os quais são de classe NP - completo. Logo, o problema estudado é no mínimo tão difícil quanto $m$ problemas independentes de classe NP completo, o que demonstra a proposição.

\subsection{Revisão Bibliográfica}

Os problemas de dimensionamento de lotes podem ser subdivididos em classes, de acordo com suas características, como por exemplo, o número de produtos (um ou vários), capacidade disponível para produção (limitada ou ilimitada), as linhas de produção (uma única linha, várias linhas paralelas, ou ainda linhas de produção dependentes), ou sobre o número de plantas (uma única planta, várias plantas). Também 
pode haver ou não relações de dependência na ordem de produção dos produtos. O problema de dimensionamento de lotes é bastante estudado na literatura, e boas revisões sobre o assunto podem ser encontradas em (Drexl \& Kimms, 1997), (Karimi, et al., 2003), (Brahimi, et al., 2006), (Jans \& Degraeve, 2008) e (Buschkühl, et al., 2010). Como ressaltado no início deste capítulo, esta revisão se restringe a detalhar apenas os problemas mais próximos do problema estudado, ou seja, problema com máquinas paralelas e problemas com plantas paralelas. Para situar o leitor historicamente, é inicialmente apresentada uma breve revisão de variantes mais simples do problema.

O tipo de problema de dimensionamento de lotes mais simples é o caso em que há a produção de um único item, em uma única máquina, com capacidade ilimitada de produção. Este problema foi estudado em (Wagner \& Within, 1958), onde são feitas constatações teóricas importantes (por exemplo, sempre existe uma solução ótima na qual a demanda é atendida totalmente pela produção do período, ou totalmente por estoque de algum dos períodos anteriores), e também é proposto um método de programação dinâmica, que resolve este problema em tempo polinomial. Este problema é muito importante, pois aparece frequentemente como subproblema de problemas mais complicados.

Em (Zangwill, 1966), é proposta uma extensão do problema, em que é permitido atender a demanda dos produtos com atraso (backlogging). $\mathrm{O}$ atraso, quando ocorre, implica em um custo adicional. São também enunciadas e demonstradas propriedades de convexidade para o problema com backlogging, e um método de programação dinâmica é proposto para resolvê-lo em tempo polinomial. Em (Zangwill, 1969), este trabalho é estendido com um modelo onde o produto pode ser processado em etapas sequenciais, ou seja, passa por diferentes processos, e só pode ser processado em uma etapa se a etapa anterior já houver sido concluída previamente. $\mathrm{O}$ autor propõe um método de programação dinâmica para resolver o problema.

Evans (1985) propôs uma codificação eficiente do método de (Wagner \& Within, 1958), no entanto, a complexidade do método não é alterada. No início dos anos 90, (Federgruen \& Tzur, 1991), (Wagelmans, et al., 1992) e (Aggarwal \& Park, 1993) propuseram, de forma independente métodos para resolver o problema com complexidade $\mathrm{O}(n \log (n))$. Boas revisões para o problema de dimensionamento de lotes de um único item, sem restrições de capacidade, podem ser encontradas em (Wosley, 1995) e (Brahimi, et al., 2006). 
A fim de aproximar os problemas estudados da realidade, novos estudos foram propostos para problemas com restrições de capacidade e múltiplos produtos. Em (Evans, 1985b) é proposto um método exato para solução do problema com múltiplos produtos, com custos de preparação de máquina e capacidade finita.

A importância dos tempos de preparação para a produção é destacada em (Trigeiro, 1987). Por ser um problema de difícil solução, poucos trabalhos da literatura abordam o problema de forma exata (Diaby, et al., 1992a); (Diaby, et al., 1992b); (Souza \& Armentano, 1994); (Armentano, et al., 1999). No entanto, várias heurísticas foram propostas para resolvê-lo como, por exemplo, as heurísticas lagrangeanas proposta por (Trigeiro, et al., 1989), (Lozano, et al., 1991) e (Hindi, 1995).

O problema de máquinas paralelas com um único produto foi proposto em (Sung, 1986). O autor monstra que para o problema com múltiplas máquinas, existe uma solução ótima tal que temos $I_{i j t} X_{i j t}=0$, para qualquer produto, qualquer máquina, e para qualquer período, ou seja, para cada produto, máquina e período, a demanda será totalmente atendida pelo estoque de algum período anterior, (e portanto não há produção), ou a demanda será totalmente atendida pela produção no período corrente em alguma máquina, e, portanto, não haverá gasto com estoque. É proposto um algoritmo de programação dinâmica para a resolução desse problema.

Poucos trabalhos da literatura tratam do problema com máquinas paralelas. Em (Carreno, 1990), é abordado o problema com máquinas paralelas idênticas. O autor estudou o problema de lote econômico e uma heurística para sua resolução. Sabbag (1993) estuda o problema de dimensionamento de lotes com múltiplos produtos, restrições de capacidade, tempos e custos de preparação de máquina. O autor propôs uma heurística construtiva para resolução do problema em que as máquinas podem ser distintas. Este mesmo problema é abordado em (Toledo \& Armentano, 2006). Os autores propõem a solução do problema utilizando uma heurística lagrangeana baseada na relaxação das restrições de capacidade do problema.

Quadt \& Kuhn (2009) confrontam o modelo clássico de dimensionamento de lotes com máquinas paralelas com um modelo adaptado proposto pelos autores. Neste artigo, também são propostas algumas heurísticas construtivas para resolver o problema.

No problema aqui estudado, são considerados múltiplos produtos, múltiplos períodos de produção, capacidade de produção limitada, e tempos e custos de preparação de máquina cada vez em que há uma troca de produtos. Além disso, é considerada a existência de múltiplas plantas, capazes de produzir todos os produtos, e é 
permitido o transporte de produtos entre as plantas. Devido à alta dificuldade intrínseca do problema estudado, os métodos encontrados na literatura para a sua resolução são todos métodos heurísticos.

Sambasivan \& Schmidt (1999) abordam o problema de dimensionamento de lotes para múltiplas plantas, múltiplos produtos, mas sem restrições de capacidade de produção. Neste trabalho, os autores discutem a abordagem do problema sem limites de capacidade de produção (assume-se que a capacidade de produção para este caso é tão grande quanto o necessário). Neste modelo, cada par "planta, período" é tratado como um nó. Os arcos representam a quantidade de produtos transportados entre plantas em um mesmo período, ou entre períodos consecutivos de uma mesma planta (ou seja, o estoque). Os autores demonstram que esta versão do problema pode ser resolvida satisfatoriamente através de um algoritmo de caminho mínimo. Uma heurística também foi proposta para o problema.

Sambasivan \& Schimidt (2002) consideram plantas com capacidade finita de produção, o que torna o problema mais complexo. Os autores propuseram um modelo para representar o problema (modelo (1) (7), descrito na Seção 2.1) e um método para sua resolução. A proposta dos autores para a resolução do problema está centrada no fato de que a ausência de restrições de capacidade permite remodelar o problema como um problema de caminho mínimo (Sambasivan \& Schmidt, 1999). Os subproblemas são resolvidos individualmente para cada produto. Caso a solução obtida respeite as restrições de capacidade, então a solução encontrada é ótima, caso contrário, tentam-se utilizar heurísticas que buscam encontrar uma solução factível para o problema. A primeira etapa dessa heurística de factibilização busca soluções factíveis fazendo trocas de produção entre plantas dentro de cada período, e a segunda etapa procura realizar trocas entre períodos de produção de uma mesma planta. Em seguida, verifica-se novamente se a solução obtida é factível, e caso não seja, a heurística é encerrada. Os autores trabalharam com um conjunto de testes com 3 ou 4 plantas, 5 ou 10 itens, e 3 ou 6 períodos, também variando as taxas de tempo de preparação de máquina, os custos de estoque e a capacidade, totalizando 256 combinações. Os resultados mostram que as soluções obtidas tem desvio máximo em relação ao valor ótimo de $2,6 \%$. Um ponto que merece destaque é o fato de que a heurística sempre obteve estas soluções em baixo tempo computacional, quando comparado ao software LINDO, chegando a ser 46 vezes mais rápida em uma das classes de teste. 
Em (Sambasivan \& Yahya, 2005), é proposta a solução desse problema utilizando uma heurística lagrangeana, na qual são relaxadas as restrições de capacidade do problema. A abordagem de solução é semelhante a (Sambasivam \& Schimidt, 2002), no entanto, nesta proposta se utiliza uma relaxação lagrangeana das restrições de capacidade com atualização dos coeficientes através do algoritmo de subgradientes. A heurística de factibilização de (Sambasivam \& Schimidt, 2002) é utilizada para obter limitantes superiores de boa qualidade para o problema. Testes computacionais utilizando 3 ou 4 plantas, 3, 4, 5 ou 6 períodos de produção e 5, 10 ou 15 produtos, num total de 24 classes diferentes foram realizados. Os resultados computacionais mostram que o desvio médio em relação à solução ótima não ultrapassa $6 \%$, e que o tempo computacional é relativamente baixo. Os autores também analisaram a influência dos parâmetros (número de plantas, produtos e períodos) em relação ao desvio e ao tempo computacional, e constataram que o aumento do número de plantas não aumenta significativamente o desvio, embora gere um aumento no tempo computacional. Em relação ao número de produtos, o desvio diminui conforme se aumenta o número de itens, embora o tempo computacional não se altere de forma significativa. E, finalmente, em relação ao número de períodos, nem o desvio médio, nem o tempo computacional variam de forma significativa.

Outro trabalho que aborda o problema de múltiplas plantas, com múltiplos itens, múltiplos períodos, capacitado, é o trabalho de (Nascimento, et al., 2010). Os autores propuseram um método GRASP com path-relinking para a resolução do problema. A abordagem consiste em, primeiramente, gerar soluções iniciais utilizando heurísticas construtivas semi-gulosas, procurando factibilizá-las caso seja possível, e realizando buscas locais nestas soluções, de modo a encontrar uma solução que seja um mínimo local para esse problema. Se uma nova solução inteira for obtida, a heurística passa para a fase de path-relinking, em que se cria um caminho entre a solução atual e a melhor solução obtida na busca, fazendo buscas locais por todo o caminho, à procura de soluções de melhor qualidade. Dois grupos de instâncias foram utilizados nos testes computacionais, o primeiro, utilizando a mesma classe de problemas apresentados em (Sambasivan \& Yahya, 2005), onde o método proposto mostrou resultados com menor desvio em praticamente todas as instâncias de teste, embora o seu tempo computacional fosse, em média, um pouco mais alto. Para esse grupo de teste, os autores constataram que a heurística GRASP com path-relinking apresentou menor desvio médio em todas as classes de teste, mas o tempo de execução foi maior, quando comparado com a 
heurística GRASP pura. Para o segundo grupo de testes, foram utilizados problemas adaptados de (Toledo \& Armentano, 2006), gerados randomicamente, onde a heurística GRASP com path-relinking novamente apresentou resultados de qualidade melhor do que a heurística GRASP pura, embora em um tempo computacional sempre maior.

Em (Özdamar \& Birbil, 1998) o problema é abordado admitindo-se que haja possibilidade de capacidade extra para a produção, com a utilização de horas-extras (overtime), o que simplifica a tarefa de obter uma solução factível inicial para o problema. Os autores tratam a versão multiestágio do problema, ou seja, a produção de alguns itens depende de outros itens componentes. Um algoritmo genético é proposto para a solução do problema. A geração da população inicial é realizada de duas maneiras: a) aleatoriamente; e b) por meio de uma combinação entre soluções aleatórias e soluções geradas por uma heurística construtiva gulosa. Outra característica interessante é a utilização de uma lista de soluções proibidas (a criação de uma lista Tabu). Testes computacionais realizados com diferentes grupos de problemas, cada qual variando o número de plantas, produtos e períodos, mostraram que os métodos híbridos que não envolvem apenas o algoritmo genético puro, ou seja, algoritmos que utilizem boas qualidades de outras heurísticas, como a lista Tabu, fornecem resultados com um GAP de otimalidade em média 1,2\% menores que o algoritmo genético puro.

Como podemos observar na literatura, o problema de dimensionamento de lotes com múltiplas plantas foi pouco estudado. Devido à sua complexidade, nenhum método exato foi proposto para sua resolução. No entanto, meta-heurísticas se mostraram boas alternativas para sua resolução. Entretanto, nenhuma math-heurística foi proposta para tratar o problema, deixando uma lacuna no estudo destes métodos. 


\section{Reformulação do Problema}

O problema de dimensionamento de lotes foi modelado na literatura de diferentes formas. Recentemente, Wu \& Shi (2011) analisaram comparativamente os principais modelos propostos. De acordo com os autores, a modelagem proposta por (Krarup \& Bilde, 1977) apresenta as melhores relaxações lineares em relação às outras, além de ser um modelo no qual métodos do tipo relax-and-fix costumam obter bons resultados computacionais para instâncias de médio porte.

Assim, para trabalhar com o problema descrito na Seção 2.1, propomos uma adaptação do modelo (1) (7), utilizando a ideia de localização de facilidades, como proposto por (Krarup \& Bilde, 1977). Embora a reformulação possua a vantagem de resultar em limitantes inferiores de melhor qualidade para o problema, o número de variáveis reais é aumentado de forma significativa, o que pode dificultar a resolução de instâncias de grande porte. Logo, neste capítulo descrevemos a reformulação proposta e analisamos sua eficiência para grupos de instâncias de teste de diferentes dimensões.

\subsection{Descrição da Reformulação}

Nesta subseção, apresentamos uma reformulação do modelo matemático (1) (7), apresentado na Seção 2.1. A ideia central da reformulação consiste em desagregar as variáveis de produção em relação às plantas e aos períodos nos quais os produtos são produzidos e entregues. Com isto, cada variável carrega consigo mais informação. Logo, as variáveis de produção que representam a quantidade produzida do produto $i$ na planta $j$ no período $t\left(X_{i j t}\right)$ são desagregadas, ou seja, as variáveis de produção passam a ser representadas por:

\footnotetext{
$X_{i j k u}$ : Quantidade do produto $i$ produzida na planta $j$, durante o período $t$, para atender a demanda (ou parte dela) da planta $k$ no período $u$.
}

A desagregação da variável de produção descarta a necessidade das variáveis de estoque, pois passamos a ter a produção diretamente direcionada a uma planta e a um período específicos, portanto, é possível saber implicitamente por quanto tempo os produtos são estocados (já que os produtos sempre serão estocados entre os períodos $t \mathrm{e}$ 
$u$ ), e, de maneira análoga, é possível saber implicitamente se haverá transporte de produtos entre plantas (haverá transporte toda vez que $X_{i j t k u} \neq 0$, para $j \neq k$ ).

Para incorporar estas mudanças, os custos de produção associados às variáveis devem englobar os custos de produção, de estoque, e de transporte de produtos entre as plantas, ou seja, o custo associado a cada variável é dado por:

$$
\bar{c}_{i j k k u}=\left\{\begin{array}{r}
0, \text { se } u<t_{;} \\
c_{i j}+r_{i j k}+(u-t) M i n\left\{h_{i j}, h_{i k}\right\}
\end{array} \quad\right. \text { caso contrário. }
$$

E o modelo original pode ser reescrito como:

$$
\text { Min } \sum_{i=1}^{n} \sum_{j=1}^{n} \sum_{t=1}^{T} \sum_{k=1}^{m} \Sigma_{t=1}^{T}\left(\bar{c}_{i j t k u} X_{i j t k u}\right)+\sum_{i=1}^{n} \sum_{j=1}^{n} \Sigma_{i=1}^{T}\left(s_{i j} Z_{i j t}\right)
$$

Sujeito à:

$$
\begin{array}{ll}
\sum_{j=1}^{m} \sum_{i=1}^{u} X_{i j t k u}=d_{i k u} & \forall i \in I, \forall k \in J, \forall u \in S_{;} \\
\sum_{i=1}^{n}\left(f_{i j} Z_{i j t}+\sum_{k=1}^{m} \sum_{u=t}^{T} b_{i j} X_{i j t k u}\right) \leq c a p_{j t} & \forall j \in J, \forall t \in S ; \\
X_{i j t k u} \leq \operatorname{Min}\left\{d_{i j t} ; \frac{c a p_{j t}-f_{j t}}{b_{j t}}\right\} & \forall i \in I, \forall j, k \in J, \forall t, u \in S_{i} \\
X_{i j t k u}=0 & \forall i \in I, \forall j, k \in J, \forall u<t \in S_{j} \\
Z_{i j t} \in\{0 ; 1\} & \forall i \in I, \forall / k \in J, \forall u \in S ; \\
X_{i j t k u} \geq 0 & \forall i \in I, \forall j, k \in J, \forall t, u \in S .
\end{array}
$$

O objetivo é minimizar o custo total de produção. A primeira parcela da função objetivo (8) contabiliza implicitamente a soma dos custos de produção, de estoque e de transporte entre plantas para todos os períodos do horizonte de produção, na segunda parcela, são contabilizados os custos de preparação de máquina. As equações (9) garantem o atendimento da demanda. As restrições (10) asseguram que a capacidade de produção é respeitada em todas as plantas em cada um dos períodos. As restrições (11) impõem que a planta esteja preparada para a produção, toda vez que houver produção do produto a ela associada. A restrição (12) é responsável por garantir que não há atrasos na entrega, e as restrições (13) (14) definem o domínio das variáveis.

Esse modelo apresenta como grande vantagem o fato de possuir uma relaxação linear mais próxima da envoltória convexa que o modelo $(1) \sim(7)$, graças aos melhores 
limitantes para as variáveis inteiras de preparação de máquina $\left(Z_{i j t}\right)$ que são limitadas pelo menor valor entre a demanda de cada período e a capacidade disponível de produção para a fabricação do produto na planta em questão, ao invés do somatório das demandas como ocorre no modelo de (Sambasivan \& Yahya, 2005). Em contrapartida, a reformulação do modelo leva ao aumento do número de variáveis de decisão e de restrições quando comparado ao modelo (1) (7). No modelo (1) (7), quando temos um problema com $n$ diferentes produtos, $m$ diferentes plantas, e $T$ períodos de produção, são necessárias $2 n m T+n m^{2} T$ variáveis reais, além de $n m T$ variáveis inteiras. Além disso, o problema apresenta $m T(4 n+n m+1)$ restrições. Já o modelo $(8) \sim(14)$, sob as mesmas condições ( $n$ itens, $m$ plantas, $T$ períodos), possui $n m^{2} T^{2}$ variáveis reais e $n m T$ variáveis inteiras, e $m T(n+n m T+1)$ restrições.

A Tabela 3.1 ilustra a relação entre as dimensões de algumas instâncias com 50 produtos para ambos os modelos. Nas três primeiras colunas são reportados, respectivamente, o número de produtos, de plantas e de períodos. Nas próximas colunas, apresentamos, respectivamente, o número de variáveis reais (VR) e o número de restrições (RE) para o modelo original e para o modelo proposto.

Tabela 3.1 - Relação entre as dimensões do problema e o número de variáveis e de restrições

\begin{tabular}{||c|c||r|r||r|r||}
\cline { 2 - 6 } \multicolumn{1}{c|}{} & \multicolumn{2}{c|}{ Modelo Original } & \multicolumn{2}{c||}{ Reformulação } \\
\hline \hline \multirow{3}{*}{12} & 2 & 4800 & 7224 & 28800 & \multicolumn{1}{c||}{30024} \\
\cline { 2 - 6 } & 4 & 14400 & 19248 & 115200 & 117648 \\
\cline { 2 - 6 } & 6 & 28800 & 36072 & 259200 & 262872 \\
\hline \multirow{3}{*}{24} & 2 & 9600 & 14448 & 115200 & 117648 \\
\cline { 2 - 6 } & 4 & 28800 & 38496 & 460800 & 465696 \\
\cline { 2 - 6 } & 6 & 57600 & 72144 & 1036800 & 1044144 \\
\hline \multirow{3}{*}{30} & 2 & 12000 & 18060 & 180000 & 183060 \\
\cline { 2 - 6 } & 4 & 36000 & 48120 & 720000 & 726120 \\
\cline { 2 - 6 } & 6 & 72000 & 90180 & 1620000 & 1629180 \\
\hline \multirow{3}{*}{45} & 2 & 18000 & 27090 & 405000 & 409590 \\
\cline { 2 - 6 } & 4 & 54000 & 72180 & 1620000 & 1629180 \\
\cline { 2 - 6 } & 6 & 108000 & 135270 & 3645000 & 3658770 \\
\hline
\end{tabular}

Como podemos observar, para o modelo reformulado o número de variáveis reais e de restrições aumenta rapidamente quando comparado ao modelo de (Sambasivan \& Yahya, 2005), ao se comparar problemas de mesma dimensão. Para a reformulação, instâncias com 30 períodos e 6 plantas já resultam em mais de um milhão 
de variáveis, ao passo que no modelo original, mesmo no maior grupo de instâncias analisadas, este valor nunca ultrapassa sequer um quarto de milhão.

Assim como para o modelo original, para o modelo (8) (14) assumimos, a princípio, que não há atrasos na entrega dos produtos, o que é garantido pela restrição (12). No entanto, este modelo também pode ser facilmente adaptado para permitir atrasos na entrega. Para isso, é necessário modificar a matriz de custos, que passa a ser:

$$
\bar{c}_{i j k k u}=\left\{\begin{array}{c}
c_{i j}+r_{i j k}+\alpha(t-u) \operatorname{Min}\left\{h_{i j}, h_{i k}\right\} \quad \text { se } u c_{i} t_{i} \\
c_{i j}+r_{i j k}+(u-t) \operatorname{Min}\left\{h_{i j} h_{i k}\right\} \text { caso contrário }
\end{array}\right.
$$

em que: $\alpha$ representa um coeficiente real $(\alpha>1)$ de peso pelo atraso na entrega. Além disso, é necessário também alterar os índices das restrições (9) e (10), que passam a ser dadas por:

$\sum_{j=1}^{m} \sum_{t=1}^{T} X_{i j \mathrm{tku}}=d_{i k u s}$

$\sum_{i=1}^{n}\left(f_{i j} Z_{i j t}+\sum_{k=1}^{m} \sum_{u=1}^{T} b_{i j} X_{i j t k u}\right) \leq c a p_{j t}$

Com essas modificações, além da remoção das restrições (12) do modelo, temos uma adaptação onde os atrasos na entrega passam a ser viáveis.

\subsection{Testes Computacionais}

Os dois modelos apresentados foram escritos e resolvidos utilizando-se o software de otimização CPLEX versão 12.4, em uma máquina com configurações Ubuntu 10.04 (64 bits), Intel Core i5 - 2300, 2.8GHz, 2Gb de RAM, com tempo limite máximo de uma hora de execução. Três classes de instâncias da literatura foram utilizadas para os testes computacionais.

A primeira classe, composta por 120 instâncias, foi proposta por (Sambasivan \& Yahya, 2005). Estas instâncias foram divididas em grupos de acordo com o número de plantas (3 ou 4), de produtos $(5,10$ ou 15) e de períodos (3, 4, 5 ou 6). A segunda classe de instâncias foi proposta por (Nascimento, et al., 2010). Esta classe é composta por 480 instâncias, divididas em 8 grupos, de acordo com os seguintes parâmetros: nivel de 
capacidade disponível $(\mathrm{A}=$ apertada; $\mathrm{N}=$ normal $)$; custo de preparação $(\mathrm{A}=$ alto; $\mathrm{B}=$ baixo); e tempo de preparação ( $\mathrm{A}=$ alto; $\mathrm{B}=$ baixo). Para cada grupo foram geradas 60 instâncias com: 2, 4 ou 6 plantas, 6, 12, 25, ou 50 produtos, e 12 períodos, sendo cinco problemas de cada dimensão. Dividindo as instâncias de acordo com os parâmetros $n, m$ e $T$, temos 12 diferentes classes de teste, cada uma com 40 instâncias. O terceiro grupo de instâncias foi adaptado de (Muller, et al., 2012). Estas instâncias originalmente foram propostas para uma única planta, com uma única máquina. Este grupo contém 300 instâncias, que foram separadas em 30 classes. Grupos com 6 produtos e 60 ou 90 períodos, com 12 produtos e 30, 45, 60 ou 90 períodos, ou com 24 produtos e 30, 45, 60 ou 90 períodos, com 2,4 ou 6 plantas, havendo 10 casos de teste para cada grupo possível.

As instâncias propostas em (Muller, et al. 2012) têm uma única planta. Para gerarmos instâncias com $m$ plantas, reproduzimos os mesmos dados da planta original $m$ vezes. No entanto, obtemos problemas com capacidade total folgada, uma vez que, na solução do problema original, haverá uma folga, na capacidade total de produção. Repetindo o problema $m$ vezes, a folga também será repetida $m$ vezes, e teremos um problema mais fácil, podendo ter economias significativas de preparação para produção. Logo, adaptamos a capacidade de cada planta como proposto em (Toledo \& Armentano, 2006).

Como desta forma a demanda para os produtos nas plantas é a mesma, também propusemos uma alteração das mesmas. Para obter a demanda de cada planta, primeiramente calculamos a demanda total de cada produto em cada período. Depois, separamos os casos para 2, 4 ou 6 plantas. Para duas plantas, é sorteado aleatoriamente um número inteiro entre 0 e 4 , temos, portanto, cinco casos possíveis, com iguais chances de ocorrer. A demanda pode estar toda concentrada em uma única planta (casos 0 e 1), pode estar distribuída numa proporção de 1:3 (casos 2 e 3), ou pode ser igual em ambas as plantas (caso 4). No caso de quatro plantas, é sorteado aleatoriamente um número inteiro entre 0 e 22, ou seja, temos 23 casos possíveis, com iguais chances de ocorrer. A demanda pode estar toda concentrada em uma única planta, pode ser dividida meio a meio entre duas plantas, pode ser dividida igualmente entre todas as plantas, ou ainda pode estar dividida entre três plantas, na proporção 1:1:2. De forma análoga, para seis plantas, temos 44 casos possíveis com iguais chances de ocorrer.

Além disso, para adaptarmos as instâncias de (Muller, et al., 2012), é necessário inserir custos de transporte entre as plantas. O custo de transporte entre plantas foi 
gerado aleatoriamente, porém com valores sorteados entre $\gamma$ e $1,9 \gamma$, onde $\gamma$ representa $o$ menor custo de estoque de um produto em qualquer planta, em qualquer período (é importante ressaltar o fato que $\gamma$ é uma constante estritamente positiva). Desta forma, independente do valor de $\gamma$ e do número de plantas, os custos de transporte gerados sempre irão satisfazer a desigualdade triangular. E sendo satisfeita a desigualdade triangular, o menor custo de transporte entre duas plantas quaisquer será sempre dado pelo transporte direto entre elas. Além disso, note que estamos assumindo que todas as plantas são ligadas entre si. O fato da desigualdade triangular ser respeitada, juntamente com o fato de que todas as plantas estarem conectadas entre si implicam em que a melhor forma de transportar qualquer produto entre plantas será sempre transportar diretamente o produto entre as plantas, e não há necessidade de resolver um problema de caminho mínimo para realizar o transporte.

\subsubsection{Análise da Relaxação Linear dos Modelos}

Primeiramente foram avaliados os limitantes obtidos ao resolvermos as relaxações lineares dos dois modelos. Nas Tabelas 3.2, 3.3 e 3.4, são reportadas as diferenças entre valores dos limitantes inferiores obtidos para as instâncias de testes nos dois modelos. Nas três primeiras colunas são definidos, respectivamente, o número de plantas, de produtos e de períodos de cada grupo de instâncias. Na quarta e quinta colunas são apresentados, respectivamente, os tempos médios para se obter uma solução ótima da relaxação linear do modelo de (Sambasivan \& Yahya, 2005) (SY) e da reformulação do problema (RP). Na última coluna da tabela apresentamos o desvio porcentual médio entre os valores ótimos das soluções em cada classe, calculado da seguinte forma:

$$
\text { Desvio }=\frac{\sum_{i=1}^{k} \frac{\left(Z_{R P_{i}}-Z_{S Y_{i}}\right) * 100}{Z_{R P_{i}}}}{k}
$$

onde:

$k$ : indica a quantidade de instâncias em um grupo de testes; 
$Z_{S Y_{i}}:$ indica o valor ótimo da relaxação linear do modelo de (Sambasivan \& Yahya, 2005) para a $i$-ésima instância da classe;

$Z_{n F_{i}}$ : indica o valor ótimo da relaxação linear do problema reformulado para a $i$-ésima instância da classe.

Para as instâncias de (Sambasivan \& Yahya, 2005), a reformulação apresentou em média um limitante de qualidade 3,8\% melhor, quando comparado à relaxação linear do modelo de (Sambasivan \& Yahya, 2005). Embora a reformulação possua um número muito maior de restrições e de variáveis, para essa classe de testes, a diferença no tempo médio de resolução dos dois modelos é inferior a um centésimo de segundo.

Tabela 3.2 - Resultados da relaxação linear dos modelos:

Instâncias de (Sambasivan e Yahya, 2005).

\begin{tabular}{|c|c|c|c|c|c|}
\hline \multirow[b]{2}{*}{ Plantas } & \multirow[b]{2}{*}{ Produtos } & \multirow[b]{2}{*}{ Períodos } & \multicolumn{2}{|c|}{ Tempo (s) } & \multirow[b]{2}{*}{$\begin{array}{c}\text { Desvio } \\
(\%)\end{array}$} \\
\hline & & & SY & $\mathbf{R P}$ & \\
\hline \multirow{12}{*}{3} & \multirow{4}{*}{5} & $3 *$ & 0,01 & 0,01 & 4,98 \\
\hline & & $4 *$ & 0,01 & 0,01 & 3,69 \\
\hline & & 5 & 0,01 & 0,01 & 4,37 \\
\hline & & 6 & 0,01 & 0,01 & 3,57 \\
\hline & \multirow{4}{*}{10} & 3 & 0,01 & 0,01 & 2,94 \\
\hline & & 4 & 0,01 & 0,01 & 3,11 \\
\hline & & 5 & 0,01 & 0,01 & 3,85 \\
\hline & & 6 & 0,01 & 0,02 & 4,16 \\
\hline & \multirow{4}{*}{15} & 3 & 0,01 & 0,02 & 3,52 \\
\hline & & 4 & 0,01 & 0,02 & 3,50 \\
\hline & & $5 *$ & 0,02 & 0,02 & 3,69 \\
\hline & & 6* & 0,02 & 0,02 & 4,06 \\
\hline \multirow{12}{*}{4} & \multirow{4}{*}{5} & 3 & 0,01 & 0,01 & 3,43 \\
\hline & & 4 & 0,01 & 0,01 & 3,91 \\
\hline & & 5 & 0,01 & 0,01 & 4,36 \\
\hline & & 6* & 0,01 & 0,02 & 4,31 \\
\hline & \multirow{4}{*}{10} & 3 & 0,01 & 0,01 & 3,19 \\
\hline & & 4 & 0,01 & 0,02 & 3,66 \\
\hline & & 5 & 0,02 & 0,02 & 3,91 \\
\hline & & 6 & 0,03 & 0,04 & 3,77 \\
\hline & \multirow{4}{*}{15} & 3 & 0,01 & 0,02 & 3,50 \\
\hline & & 4 & 0,02 & 0,02 & 4,02 \\
\hline & & 5 & 0,04 & 0,05 & 3,56 \\
\hline & & 6 & 0,05 & 0,06 & 4,13 \\
\hline \multicolumn{3}{|c|}{ Média } & 0,02 & 0,02 & $3, \mathbf{3 0}$ \\
\hline
\end{tabular}

*De acordo com os dados, estes grupos possuem apenas quatro instâncias ao invés de cinco. 
Para a segunda classe de instâncias, notamos uma melhora média de 10,82\% no valor do limitante encontrado na relaxação linear quando a reformulação é confrontada com o modelo de (Sambasivan \& Yahya, 2005). Além disso, a diferença entre os tempos médios necessários para se obter o ótimo do problema relaxado ainda é inferior a um centésimo de segundo.

Tabela 3.3 - Resultados da relaxação linear dos modelos:

Instâncias de (Nascimento, Resende, e Toledo, 2010).

\begin{tabular}{|c|c|c|c|c|c|}
\hline \multirow[b]{2}{*}{ Plantas } & \multirow[b]{2}{*}{ Produtos } & \multirow[b]{2}{*}{ Períodos } & \multicolumn{2}{|c|}{ Tempo (s) } & \multirow[b]{2}{*}{$\begin{array}{c}\text { Desvio } \\
(\%)\end{array}$} \\
\hline & & & SY & $\mathbf{R P}$ & \\
\hline \multirow{4}{*}{2} & 6 & \multirow{4}{*}{12} & 0,01 & 0,01 & 12,52 \\
\hline & 12 & & 0,01 & 0,01 & 11,47 \\
\hline & 25 & & 0,02 & 0,02 & 11,95 \\
\hline & 50 & & 0,01 & 0,01 & 12,17 \\
\hline \multirow{4}{*}{4} & 6 & \multirow{4}{*}{12} & 0,02 & 0,02 & 11,24 \\
\hline & 12 & & 0,04 & 0,04 & 10,54 \\
\hline & 25 & & 0,03 & 0,03 & 10,79 \\
\hline & 50 & & 0,05 & 0,06 & 11,72 \\
\hline \multirow{4}{*}{6} & 6 & \multirow{4}{*}{12} & 0,09 & 0,10 & 9,18 \\
\hline & 12 & & 0,06 & 0,05 & 9,42 \\
\hline & 25 & & 0,10 & 0,10 & 9,45 \\
\hline & 50 & & 0,21 & 0,20 & 9,37 \\
\hline \multicolumn{3}{|c|}{ Média } & 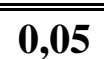 & $\overline{0.05}$ & $\begin{array}{l}10,82 \\
\end{array}$ \\
\hline
\end{tabular}

Finalmente, para a terceira classe de instâncias, devido ao grande número de variáveis da reformulação, algumas instâncias não puderam ser resolvidas por falta de memória. No entanto, para o modelo de (Sambasivan \& Yahya, 2005), todas as relaxações lineares dos problemas foram resolvidas. Para as instâncias em que ambos os modelos apresentam solução, a reformulação do problema resulta numa relaxação linear de melhor qualidade.

A Tabela 3.4 apresenta uma coluna a mais que as Tabelas 3.2 e 3.3, para representar o número de instâncias dentro de cada grupo que foram resolvidas até a otimalidade em sua relaxação linear, pela reformulação do modelo (NP). Lembramos que em cada classe de testes há 10 problemas. Na Tabela 3.4, a expressão “--" significa que nenhuma instância foi resolvida, logo o Desvio não pôde ser calculado. 
Tabela 3.4 - Resultados da relaxação linear dos modelos:

Instâncias de (Muller, et al., 2012).

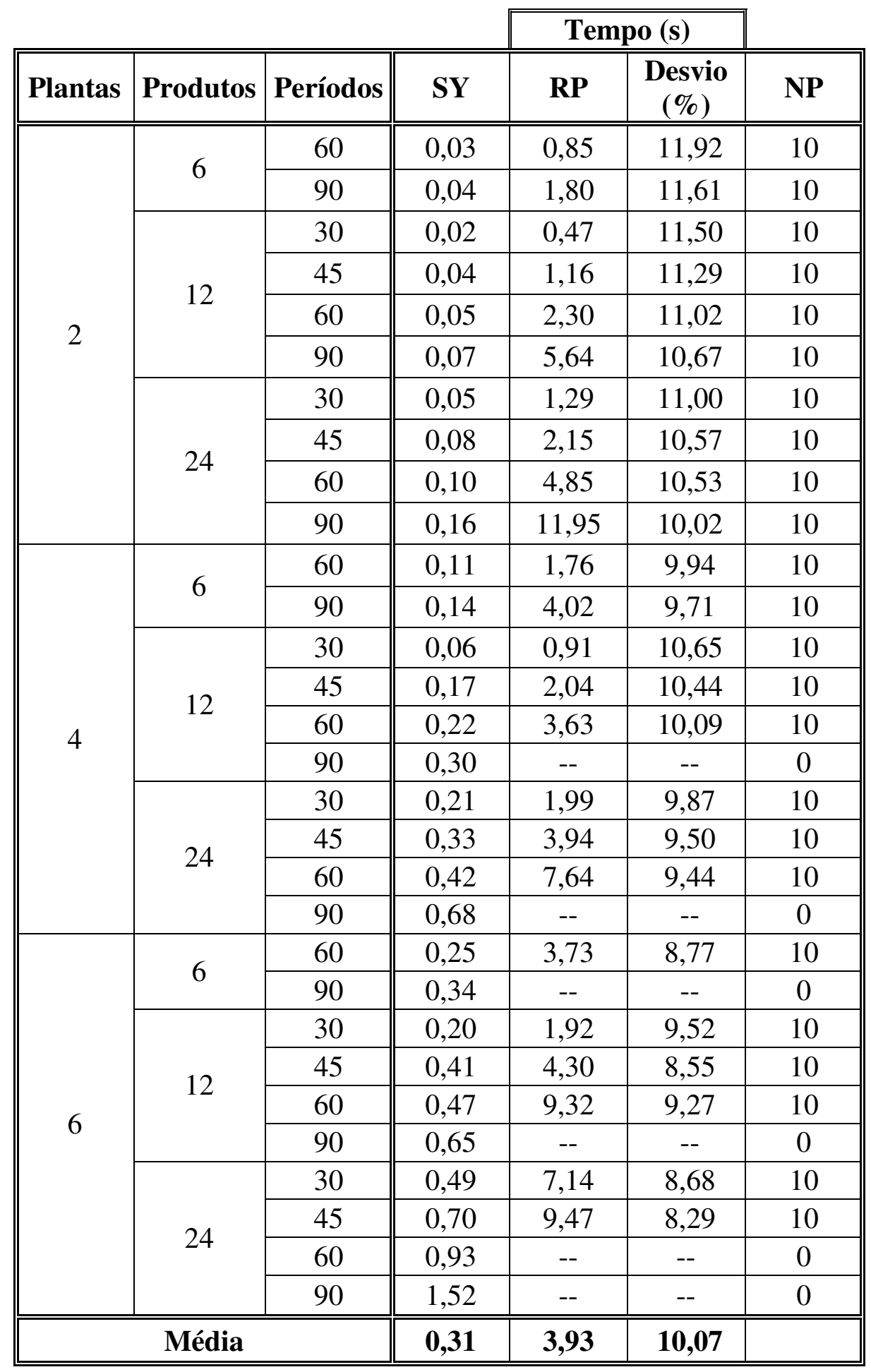


Assim como para as outras instâncias, a relaxação linear da reformulação apresentou uma qualidade melhor que a relaxação linear do modelo de (Sambasivan \& Yahya, 2005). Embora o tempo de execução para a relaxação linear do problema pela reformulação proposta seja em média 12,68 vezes maior, ele ainda é baixo em valor absoluto, sempre abaixo de um minuto, nos casos em que a relaxação linear é resolvida.

Foram realizados testes admitindo-se a possibilidade de atrasos na entrega (backlogging), utilizando o coeficiente $\alpha=1,5$ na matriz de custos. As Tabelas 3.5, 3.6 e 3.7 resumem os resultados obtidos para os três grupos de instâncias teste.

Tabela 3.5 - Resultados da relaxação linear para o problema com possibilidade de atrasos na entrega: Instâncias de (Sambasivan \& Yahya, 2005).

\begin{tabular}{|c|c|c|c|c|c|}
\hline \multirow[b]{2}{*}{ Plantas } & \multirow[b]{2}{*}{ Produtos } & \multirow[b]{2}{*}{ Períodos } & \multicolumn{2}{|c|}{ Tempo (s) } & \multirow[b]{2}{*}{$\begin{array}{c}\text { Desvio } \\
(\%) \\
\end{array}$} \\
\hline & & & SY & $\mathbf{R P}$ & \\
\hline \multirow{12}{*}{3} & \multirow{4}{*}{5} & $3 *$ & 0,01 & 0,01 & 4,92 \\
\hline & & $4 *$ & 0,01 & 0,01 & 3,64 \\
\hline & & 5 & 0,01 & 0,01 & 4,37 \\
\hline & & 6 & 0,01 & 0,01 & 3,54 \\
\hline & \multirow{4}{*}{10} & 3 & 0,01 & 0,01 & 2,91 \\
\hline & & 4 & 0,01 & 0,01 & 3,08 \\
\hline & & 5 & 0,01 & 0,01 & 3,82 \\
\hline & & 6 & 0,01 & 0,02 & 4,11 \\
\hline & \multirow{4}{*}{15} & 3 & 0,01 & 0,02 & 3,46 \\
\hline & & 4 & 0,01 & 0,02 & 3,44 \\
\hline & & $5 *$ & 0,02 & 0,02 & 3,63 \\
\hline & & 6* & 0,02 & 0,02 & 4,02 \\
\hline \multirow{12}{*}{4} & \multirow{4}{*}{5} & 3 & 0,01 & 0,01 & 3,37 \\
\hline & & 4 & 0,01 & 0,01 & 3,84 \\
\hline & & 5 & 0,01 & 0,01 & 4,29 \\
\hline & & $6 *$ & 0,01 & 0,02 & 4,23 \\
\hline & \multirow{4}{*}{10} & 3 & 0,01 & 0,01 & 3,14 \\
\hline & & 4 & 0,01 & 0,02 & 3,60 \\
\hline & & 5 & 0,02 & 0,02 & 3,84 \\
\hline & & 6 & 0,03 & 0,04 & 3,71 \\
\hline & \multirow{4}{*}{15} & 3 & 0,01 & 0,01 & 3,47 \\
\hline & & 4 & 0,02 & 0,02 & 3,98 \\
\hline & & 5 & 0,04 & 0,05 & 3,53 \\
\hline & & 6 & 0,05 & 0,05 & 4,06 \\
\hline \multicolumn{3}{|c|}{ Média } & 0,02 & 0,02 & 3,75 \\
\hline
\end{tabular}

* De acordo com os dados, estes grupos possuem apenas quatro instâncias ao invés de cinco. 
Tabela 3.6 - Resultados da relaxação linear para o problema com possibilidade de atrasos na entrega:

Instâncias de Nascimento, Resende e Toledo (2010).

\begin{tabular}{|c|c|c|c|c|c|}
\hline \multirow[b]{2}{*}{ Plantas } & \multirow[b]{2}{*}{ Produtos } & \multirow[b]{2}{*}{ Períodos } & \multicolumn{2}{|c|}{ Tempo (s) } & \multirow[b]{2}{*}{$\begin{array}{c}\text { Desvio } \\
(\%) \\
\end{array}$} \\
\hline & & & SY & $\mathbf{R P}$ & \\
\hline \multirow{4}{*}{2} & 6 & \multirow{4}{*}{12} & 0,01 & 0,00 & 12,03 \\
\hline & 12 & & 0,02 & 0,01 & 11,05 \\
\hline & 25 & & 0,02 & 0,02 & 11,47 \\
\hline & 50 & & 0,01 & 0,01 & 11,69 \\
\hline \multirow{4}{*}{4} & 6 & \multirow{4}{*}{12} & 0,02 & 0,02 & 10,04 \\
\hline & 12 & & 0,04 & 0,04 & 10,16 \\
\hline & 25 & & 0,03 & 0,03 & 10,36 \\
\hline & 50 & & 0,05 & 0,05 & 10,49 \\
\hline \multirow{4}{*}{6} & 6 & \multirow{4}{*}{12} & 0,10 & 0,10 & 8,82 \\
\hline & 12 & & 0,06 & 0,05 & 9,09 \\
\hline & 25 & & 0,10 & 0,09 & 9,13 \\
\hline & 50 & & 0,22 & 0,19 & 9,04 \\
\hline \multicolumn{3}{|c|}{ Plantas } & 0,06 & 0,05 & 10,28 \\
\hline
\end{tabular}

Podemos observar nas Tabelas de 3.2 à 3.7 que, para as duas primeiras classes de instâncias, o tempo médio de resolução das relaxações lineares dos problemas com possibilidade de atraso na entrega é aproximadamente o mesmo, mas na terceira classe de testes o tempo médio de resolução para a relaxação linear pelo modelo de (Sambasivan \& Yahya, 2005) é significativamente menor do que o tempo para a reformulação proposta. Ainda assim, o tempo de resolução para todos os testes (nos quais os problemas foram resolvidos) é sempre inferior a um minuto.

Notamos também que para as três classes a melhoria média do limitante é semelhante as instâncias em que não é permitido a atraso na entrega da demanda. Como última observação, notamos que os problemas que não puderam ser resolvidos sem admitir possibilidade de atrasos na entrega também não foram resolvidos. 
Tabela 3.7 - Resultados da relaxação linear para o problema com possibilidade de atrasos na entrega: Instâncias de (Muller, et al., 2012).

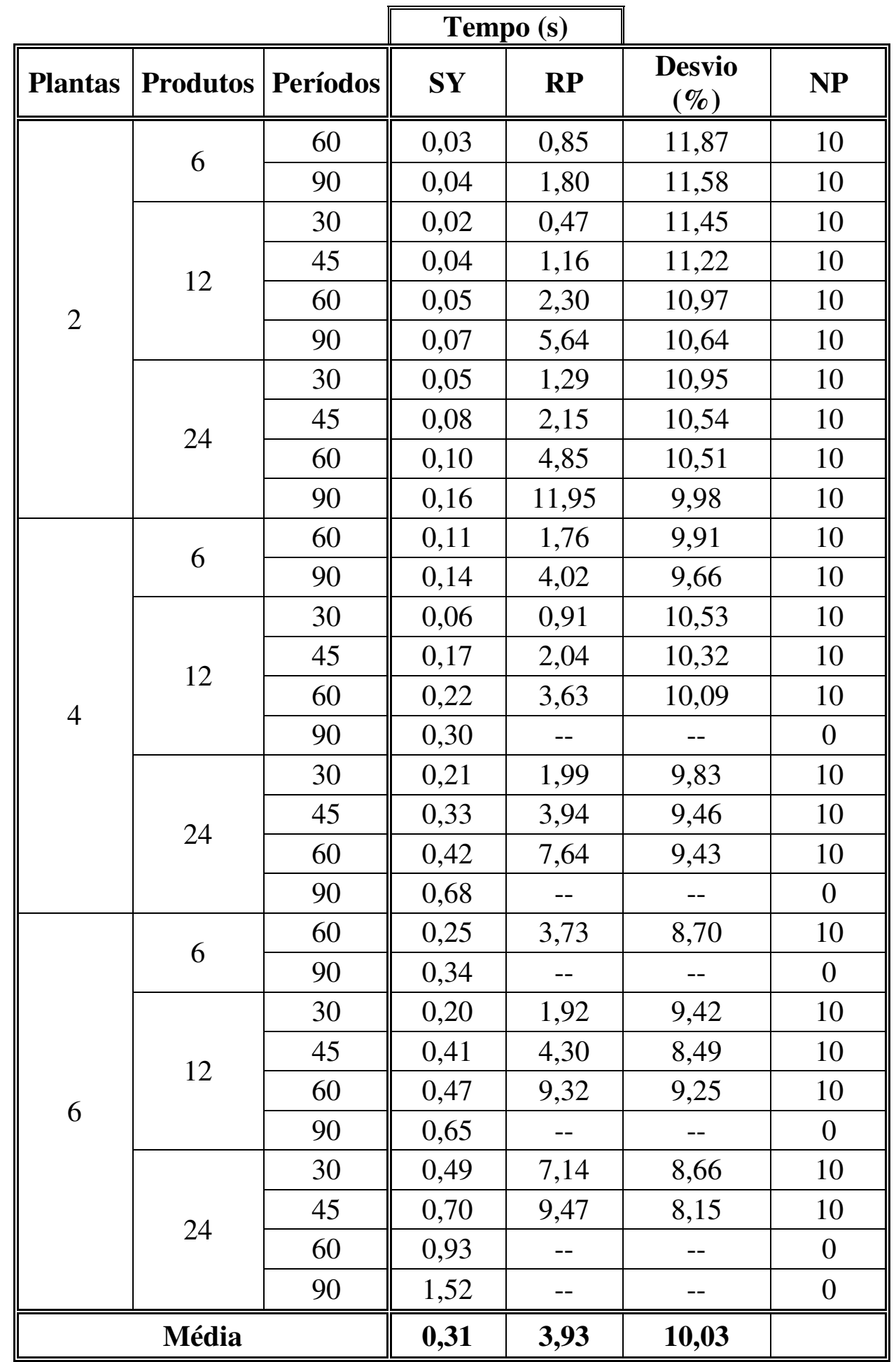




\subsubsection{Análise do Desempenho do Programa Inteiro Misto}

Para os problemas inteiro-mistos, as instâncias anteriores foram resolvidas utilizando o software de otimização CPLEX, com tempo limite máximo de uma hora de execução. Todas as instâncias da primeira classe foram resolvidas por ambos os modelos em menos de 10 segundos cada. Já para a segunda classe, o modelo proposto se mostrou mais eficiente, como destacado na Tabela 3.8. Para a terceira classe de testes, o modelo proposto teve bom desempenho nas instâncias de menor dimensão, mas não encontrou uma solução ótima para os problemas de maior dimensão.

Na Tabela 3.8, as três primeiras colunas definem, respectivamente, o número de plantas, o número de produtos e o número de períodos de cada classe de testes, para as instâncias de (Nascimento, et al., 2010). Nas quarta e quinta colunas são apresentados, respectivamente, os tempos médios em segundos para se obter uma solução ótima utilizando o modelo de (Sambasivan \& Yahya, 2005) (TM), as instâncias para as quais uma solução ótima foi obtida antes de atingir o tempo limite (uma hora), e o número de problemas que foram resolvidos na otimalidade em cada grupo (NO). Nas sexta e sétima colunas são apresentados, os mesmos dados para a reformulação do modelo.

Tabela 3.8 - Resultados para o problema inteiro misto:

Instâncias de (Nascimento, et al., 2010).

\begin{tabular}{|c|c|c|c|c|c|c|}
\hline \multirow[b]{2}{*}{ Plantas } & \multirow[b]{2}{*}{ Produtos } & \multirow[b]{2}{*}{ Períodos } & \multicolumn{2}{|c|}{ SY } & \multicolumn{2}{|c|}{ RP } \\
\hline & & & TM (s) & NO & TM (s) & NO \\
\hline \multirow{4}{*}{2} & 6 & \multirow{4}{*}{12} & 1,34 & 40 & 0,04 & 40 \\
\hline & 12 & & 330,39 & 39 & 0,08 & 40 \\
\hline & 25 & & 63,78 & 16 & 0,20 & 40 \\
\hline & 50 & & -- & 0 & 0,52 & 40 \\
\hline \multirow{4}{*}{4} & 6 & \multirow{4}{*}{12} & 341,14 & 37 & 0,18 & 40 \\
\hline & 12 & & 29,71 & 20 & 0,39 & 40 \\
\hline & 25 & & 125,66 & 20 & 1,16 & 40 \\
\hline & 50 & & 150,19 & 18 & 2,51 & 40 \\
\hline \multirow{4}{*}{6} & 6 & \multirow{4}{*}{12} & 60,02 & 18 & 0,43 & 40 \\
\hline & 12 & & 12,08 & 20 & 1,09 & 40 \\
\hline & 25 & & 8,22 & 20 & 3,34 & 40 \\
\hline & 50 & & 253,18 & 20 & 9,23 & 40 \\
\hline \multicolumn{3}{|c|}{ "Totais } & & 268 & & 480 \\
\hline
\end{tabular}


Utilizando a reformulação proposta foi possível obter uma solução ótima para todas as instâncias testes com tempo médio igual a 1,6 segundos. Destacamos ainda que cada uma das instâncias teste foi resolvida em menos de 30 segundos. Utilizando o modelo original foram obtidas soluções ótimas para apenas 268 instâncias antes de atingir o tempo limite, ou seja, aproximadamente 56\% das instâncias. Além disso, o tempo médio para resolver as instâncias teste (que não foram encerradas por atingir o tempo limite) foi de 108,7 segundos; logo, a reformulação é mais vantajosa para solução destas instâncias.

Na Tabela 3.9 são apresentados os resultados obtidos para as instâncias de (Muller, et al., 2012). Nesta tabela, as três primeiras colunas apresentam as dimensões do problema. As colunas quatro, cinco e seis são referentes a modelagem apresentada em (Sambasivan \&, Yahya, 2005). Na quarta é reportado o tempo médio para se obter uma solução ótima para cada classe de instâncias. Este tempo foi calculado considerando apenas as instâncias em que uma solução ótima foi obtida antes de atingir o tempo limite. Na quinta e na sexta colunas, são apresentados o número de instâncias para as quais uma solução ótima foi obtida (NO) e o número de instâncias para as quais uma solução factível foi obtida (NF). Estes mesmos dados são apresentados na sétima, oitava e nona colunas, para a reformulação do modelo.

Observamos que a reformulação do modelo não é capaz de resolver na otimalidade todas as instâncias de teste. Foram resolvidos na otimalidade apenas 100 das 300 instâncias $(33,33 \%)$ e foi encontrada pelo menos uma solução factível para 54\% das instâncias. Para as instâncias grandes ( 24 produtos, 4 plantas e 60 e 90 períodos, e 12 e 24 produtos, 6 plantas, 60 e 90 períodos), por falta de memória, não foi possível resolver nem a relaxação linear. Além disso, para a classe de instâncias com 2 plantas, 24 produtos e 30 períodos, a reformulação não determinou nenhuma solução ótima, mesmo a memória sendo suficiente para ler os dados do programa.

Embora utilizando o modelo proposto em (Sambasivan \& Yahya, 2005) não seja viável encontrar solução ótima para nenhuma das instâncias no tempo limite de uma hora, foi possível obter soluções factíveis para $89 \%$ das instâncias, 35\% a mais que utilizando a reformulação. Esta diferença é mais significativa para as instâncias de maior dimensão.

As Tabelas 3.10 e 3.11 mostram os resultados obtidos para os mesmos problemas, mas no caso em que admitimos possibilidade de atrasos na entrega, utilizando o coeficiente $\alpha=1,5$, também utilizado nos testes para as relaxações lineares. 
Tabela 3.9 - Resultados para o problema inteiro misto:

Instâncias de (Muller, et al., 2012).

\begin{tabular}{|c|c|c|c|c|c|c|c|c|}
\hline & & & & SY & & & $\mathbf{R P}$ & \\
\hline Plantas & Produtos & Períodos & TM (s) & NO & NF & TM (s) & NO & NF \\
\hline & 6 & 60 & -- & 0 & 6 & 2580,27 & 3 & 9 \\
\hline & 0 & 90 & -- & 0 & 10 & 2043,97 & 3 & 7 \\
\hline & & 30 & -- & 0 & 6 & 2016,99 & 1 & 9 \\
\hline & & 45 & -- & 0 & 8 & 2799,29 & 2 & 10 \\
\hline 0 & 12 & 60 & -- & 0 & 10 & 1900,29 & 3 & 9 \\
\hline 2 & & 90 & -- & 0 & 10 & 2966,20 & 2 & 7 \\
\hline & & 30 & -- & 0 & 10 & -- & 0 & 5 \\
\hline & 21 & 45 & -- & 0 & 8 & 2179,22 & 5 & 7 \\
\hline & 24 & 60 & -- & 0 & 7 & 2550,34 & 6 & 7 \\
\hline & & 90 & -- & 0 & 6 & 2420,28 & 6 & 9 \\
\hline & 6 & 60 & -- & 0 & 10 & 1291,93 & 6 & 9 \\
\hline & 0 & 90 & -- & 0 & 9 & 1257,14 & 7 & 7 \\
\hline & & 30 & -- & 0 & 10 & 1369,23 & 2 & 4 \\
\hline & 12 & 45 & -- & 0 & 7 & 635,61 & 8 & 9 \\
\hline 1 & 12 & 60 & -- & 0 & 7 & 2560,57 & 1 & 1 \\
\hline 4 & & 90 & -- & 0 & 9 & 1158,51 & 8 & 8 \\
\hline & & 30 & -- & 0 & 9 & 357,39 & 5 & 7 \\
\hline & 24 & 45 & -- & 0 & 10 & 523,38 & 9 & 9 \\
\hline & 24 & 60 & -- & 0 & 9 & -- & 0 & 0 \\
\hline & & 90 & -- & 0 & 10 & -- & 0 & 0 \\
\hline & 6 & 60 & -- & 0 & 10 & 2080,26 & 1 & 2 \\
\hline & 0 & 90 & -- & 0 & 10 & 118,80 & 3 & 4 \\
\hline & & 30 & -- & 0 & 10 & 193,58 & 3 & 4 \\
\hline & 12 & 45 & -- & 0 & 10 & 105,93 & 8 & 10 \\
\hline 6 & 12 & 60 & -- & 0 & 9 & -- & 0 & 0 \\
\hline 0 & & 90 & -- & 0 & 10 & -- & 0 & 0 \\
\hline & & 30 & -- & 0 & 8 & 633,90 & 4 & 4 \\
\hline & 24 & 45 & -- & 0 & 9 & 785,94 & 4 & 4 \\
\hline & 24 & 60 & -- & 0 & 10 & -- & 0 & 0 \\
\hline & & 90 & -- & 0 & 10 & -- & 0 & 0 \\
\hline & Total & & & 0 & 267 & & 100 & 161 \\
\hline
\end{tabular}


Tabela 3.10 - Resultados do modelo inteiro misto com possibilidade de atraso na entrega: Instâncias de (Nascimento, et al., 2010).

\begin{tabular}{|c|c|c|c|c|c|c|}
\hline \multirow[b]{2}{*}{ Plantas } & \multirow[b]{2}{*}{ Produtos } & \multirow[b]{2}{*}{ Períodos } & \multicolumn{2}{|c|}{ SY } & \multicolumn{2}{|c|}{$\mathbf{R P}$} \\
\hline & & & TM (s) & NO & TM (s) & NO \\
\hline \multirow{4}{*}{2} & 6 & \multirow{4}{*}{12} & 1,34 & 40 & 0,06 & 40 \\
\hline & 12 & & 330,39 & 39 & 0,36 & 40 \\
\hline & 25 & & 63,78 & 16 & 0,54 & 40 \\
\hline & 50 & & -- & 0 & 1,20 & 40 \\
\hline \multirow{4}{*}{4} & 6 & \multirow{4}{*}{12} & 341,14 & 37 & 0,36 & 40 \\
\hline & 12 & & 29,71 & 20 & 1,38 & 40 \\
\hline & 25 & & 125,66 & 20 & 1,98 & 40 \\
\hline & 50 & & 150,19 & 18 & 4,26 & 40 \\
\hline \multirow{4}{*}{6} & 6 & \multirow{4}{*}{12} & 60,02 & 28 & 0,84 & 40 \\
\hline & 12 & & 12,08 & 20 & 2,94 & 40 \\
\hline & 25 & & 8,22 & 20 & 3,60 & 40 \\
\hline & 50 & & 253,18 & 20 & 9,06 & 40 \\
\hline \multicolumn{3}{|c|}{ Totais } & & 278 & & 480 \\
\hline
\end{tabular}

Podemos observar que o modelo proposto também apresenta resultados muito satisfatórios quando resolvemos o mesmo problema admitindo possibilidade de atrasos na entrega de produtos. Embora o tempo médio de resolução dos problemas seja maior que o tempo médio de resolução dos problemas sem possibilidade de atrasos na entrega, a reformulação se mostra muito rápida. Destacamos também o fato de que todas as 480 instâncias são resolvidas, já para o modelo de (Sambasivan \& Yahya, 2005) foram resolvidas apenas 278 das 480 instâncias, representando aproximadamente $58 \%$ do total, um valor $2 \%$ maior do que o apresentado para o problema sem considerar a possibilidade de atraso na entrega. Outro ponto que merece destaque é o fato de que utilizando a reformulação, para todas as instâncias foi encontrada uma solução ótima para o problema em tempos sempre inferiores à 40 segundos, independente de considerarmos a possibilidade de atrasos na entrega ou não, ou seja, utilizando a reformulação, estas instâncias são facilmente resolvidas. 
Tabela 3.11 - Resultados do modelo inteiro misto com possibilidade de atraso na entrega: Instâncias de (Muller, et al., 2012).



Para as instâncias de (Muller, et al, 2012), quando há possibilidade de atrasos na entrega, a reformulação do modelo comportou-se de forma semelhante ao caso sem possibilidade de atrasos na entrega. A reformulação encontrou uma solução ótima dentro do tempo limite de execução em 113 das 300 instâncias, ou seja, 
aproximadamente 37,67\% dos casos testados. Além disso, foram determinadas soluções factíveis dentro do tempo de execução em 174 instâncias, que representam um total de 58\% das instâncias. Novamente, a reformulação saiu-se melhor nas instâncias de porte médio, resolvendo um número maior de problemas até a otimalidade, e em um tempo menor. Além disso, é possível notar que pelo modelo de (Sambasivan \& Yahya, 2005), nenhuma instância foi resolvida até a otimalidade, embora tenham sido encontradas soluções factíveis dentro do tempo limite de execução para 96,33\% das instâncias. Para ambos os modelos, para o problema admitindo possibilidade de atraso na entrega foi possível encontrar soluções ótimas e factíveis em maior número, como era esperado.

\subsection{Conclusões}

Neste capítulo foi proposta uma reformulação do modelo matemático para dimensionamento de lotes com múltiplas plantas, múltiplos produtos, múltiplos períodos, capacidade limitada e considerando tempo de preparação de máquina, proposto originalmente em (Sambasivan \& Yahya, 2005). A remodelagem envolve a ideia do modelo de localização de facilidades, que desagrega as variáveis de produção, como proposto em (Krarup \& Bilde, 1977).

Analisamos o modelo proposto em duas etapas. Primeiramente resolvemos três diferentes grupos de instâncias testes, duas da literatura, e uma adaptada da literatura, e utilizamos suas relaxações lineares para compararmos a qualidade dos limitantes obtidos, bem como o tempo necessário para obtê-los. Em seguida, resolvemos as mesmas instâncias para os problemas inteiro-mistos correspondentes, e comparamos a qualidade das soluções inteiras obtidas no tempo limite de uma hora.

Embora o modelo proposto tenha um número de restrições e de variáveis maior que o modelo original, a partir da análise de resultados obtidos, concluímos que este apresenta melhores resultados em sua relaxação linear quando comparado ao modelo original, e além disso, que pôde ser resolvido mais rapidamente que o modelo original, quando são comparadas as resoluções dos problemas inteiro-mistos.

Por outro lado, devido ao aumento do número de variáveis e de restrições do problema no modelo proposto, existem instâncias que não podem ser tratadas computacionalmente por este modelo, ao passo que elas podem ser tratadas pelo modelo 
original, mostrando que a reformulação, embora apresente bons resultados, é limitada em relação ao tamanho das instâncias que podem ser resolvidas.

Concluímos então que, como reportado na literatura para outros problemas de dimensionamento de lotes (Wu \& Shi, 2011), a modelagem utilizando a ideia de localização de facilidades também é eficiente para o problema com múltiplas plantas, mas é mais limitada em relação ao tamanho das instâncias que podem ser tratadas por essa modelagem. Por isso, vamos propor no Capítulo 4 uma math-heurística para tratar este problema para instâncias de grande magnitude. 


\section{Um Método Híbrido}

A reformulação do problema descrita no Capítulo 3, embora apresente bons resultados para as instâncias menores, torna-se inviável para as instâncias de maiores dimensões, uma vez que, devido ao grande número de variáveis e restrições que utilizadas na remodelagem, as instâncias de maiores dimensões não puderem sequer ser lidas pelo software CPLEX. Logo, o método híbrido (math-heurística) proposto neste capítulo foi baseado no modelo de Sambasivan e Yahya (2005).

Nosso objetivo é propor um método híbrido (math-heurística) para resolver o problema de dimensionamento de lotes estudado. A abordagem proposta combina o método relax-and-fix apresentado em (Dillenberger, et al., 1994) com a restrição de local branching proposta por Fischetti e Lodi (2003). Logo, nas Seções 4.1 e 4.2, resumimos, respectivamente, o método relax-and-fix e a restrição de local branching. $\mathrm{Na}$ Seção 4.3, descrevemos a math-heurística proposta, enquanto na Seção 4.4, apresentamos os testes computacionais realizados para avaliá-la.

\subsection{Método Relax-and-fix}

Sabemos que, para problemas de otimização inteiro-mistos, a dificuldade de sua resolução está associada as suas variáveis inteiras. Quanto maior o número de variáveis inteiras, mais difícil sua resolução. O método relax-and-fix consiste, basicamente, na resolução iterativa de um problema, através de sua decomposição em subproblemas menores, cada um deles com um número menor de variáveis inteiras (Dillenberger, et al., 1994).

Para aplicar o método relax-and-fix, as variáveis inteiras são divididas em três grupos:

i) Variáveis inteiras com valor fixado;

ii) Variáveis inteiras não-fixadas, mas relaxadas linearmente;

iii) Variáveis inteiras não-fixadas inteiras;

Em sua versão mais simples, no primeiro passo do método, considera-se dentre as variáveis inteiras, uma partição das variáveis como sendo inteiras, e as demais são consideradas linearmente relaxadas. Resolve-se então um subproblema inteiro-misto (que é de resolução mais fácil que o problema inteiro-misto original, dado que o número 
de variáveis inteiras é menor). As variáveis inteiras obtidas na resolução do problema são fixadas, e então um novo grupo de variáveis inteiras até então relaxadas passam a ser considerado inteiro. Repete-se o processo, até que todas as variáveis inteiras estejam fixadas, e uma solução para o problema original seja obtida.

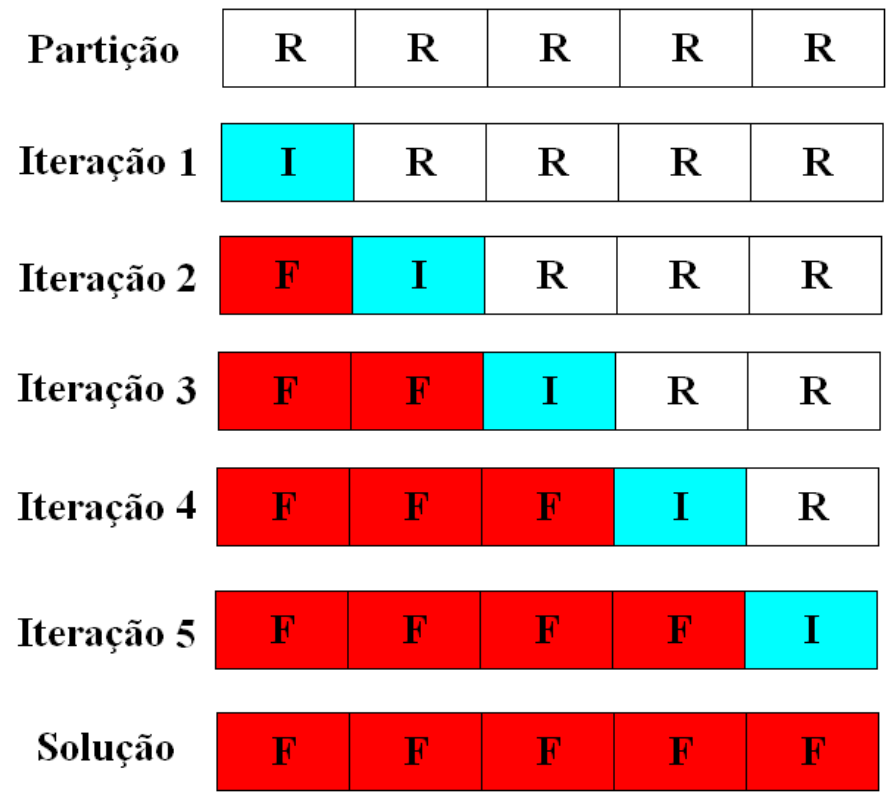

Figura 4.1 - Representação esquemática do método Relax-and-fix.

A Figura 4.1 ilustra esquematicamente a ideia do método relax-and-fix. Cada linha representa todas as variáveis inteiras do problema original, e cada retângulo menor representa uma partição destas variáveis. Os retângulos vermelhos, indicados com a letra F, representam variáveis inteiras fixadas, os retângulos brancos, indicados com a letra $\mathbf{R}$, representam variáveis inteiras relaxadas linearmente, e os retângulos azuis, indicados com a letra $\mathbf{I}$, representam variáveis inteiras. Em cada iteração do método, é resolvido um subproblema, com uma quantidade reduzida de variáveis inteiras, os valores dessas variáveis são então fixados, e uma próxima partição das variáveis passa de relaxada a inteira. A cada iteração um novo subproblema é resolvido. O método termina quando não é possível obter uma solução para um subproblema, ou seja, quando este é infactível, ou quando o subproblema associado a última partição for resolvido. Se o método resultar numa solução factível, esta é também solução do problema original.

Um dos pontos importantes do método é a escolha das partições das variáveis inteiras. Outro ponto importante é a escolha de quantas variáveis inteiras serão fixadas 
em cada iteração, já que, quanto mais variáveis inteiras, maior o tempo de execução de cada subproblema.

\subsection{Restrição de Local Branching}

Dado um problema de otimização inteiro, ou inteiro misto, do qual se conhece uma solução não necessariamente ótima, a restrição de local branching (Fischetti \& Lodi, 2003) tem como objetivo a busca de uma solução factível de melhor qualidade na vizinhança desta solução. Esta vizinhança é definida por todas as soluções vizinhas da solução atual que contenham no máximo $\mathrm{C}_{\mathrm{LB}}$ variáveis inteiras com valores diferentes da solução atual, ou seja, dada uma solução s, a restrição de local branching é dada por:

$$
\sum_{\mathrm{z}_{\mathrm{j} \mathrm{j}} \in \mathrm{Z}^{0}} \mathrm{Z}_{\mathrm{ij \textrm {t }}}+\sum_{\mathrm{z}_{\mathrm{j} \mathrm{j}}=\mathrm{Z}^{1}}\left(1-\mathrm{z}_{\mathrm{ij} \mathrm{t}}\right) \leq \mathrm{C}_{\mathrm{LE}}
$$

em que:

$\mathrm{Z}^{0}$ : indica o conjunto das variáveis binárias cujo valor é zero em s;

$\mathrm{Z}^{1}$ : indica o conjunto das variáveis binárias cujo valor é um em s;

$\mathrm{C}_{\mathrm{LB}}$ : é o limite de variáveis binárias que podem ter seu valor alterado.

O primeiro somatório indica a soma de todas as variáveis inteiras, cujo valor é zero na solução s, ou seja, caso numa solução vizinha a uma destas variáveis assuma o valor um, ela será contabilizado nesta somatória. Por outro lado, o segundo somatório contabiliza as variáveis que eram um e que tiverem seu valor alterado para zero serão contabilizadas. Logo, a soma destes dois somatórios resulta no número de variáveis cujos valores foram alterados, que é limitado a $C_{L B}$. Vale destacar que caso em que $C_{L B}$ seja igual a zero, todas as variáveis binárias não podem ter seu valor alterado, ou seja, estão fixas.

Quando adicionamos essa restrição ao problema original e resolvemos o subproblema resultante, buscamos encontrar uma solução vizinha de $\mathrm{s}$ de melhor qualidade, ou seja, a solução ótima na vizinhança de s. 
Como podemos observar, a escolha do parâmetro $\mathrm{C}_{\mathrm{LB}}$ é muito importante, pois valores muito grandes, ampliam a vizinhança e podem tornar o subproblema resultante difícil de resolver, embora, em geral, levem a soluções de melhor qualidade. Por outro lado, valores pequenos de $\mathrm{C}_{\mathrm{LB}}$, resultam em problemas mais fáceis de resolver, com menor vizinhança, e, portanto, com menores chances de melhorias significativas em relação a qualidade da solução atual.

\subsection{Método Proposto}

O método híbrido (math-heurística) proposto tem como base o método relaxand-fix. Este método foi utilizado com sucesso para resolver diferentes problemas de dimensionamento de lotes, como, por exemplo: (Toso, et al., 2009), (Bertaldi, et al., 2008) e (Mohammadi, et al., 2010).

Para o problema estudado, temos variáveis inteiras de preparação para a produção (setup), indicadas no modelo como $Z_{i j t}$, totalizando n.m.T variáveis inteiras (onde $\mathrm{n}$ é número de diferentes produtos produzidos, $\mathrm{m}$ é o número de plantas, e $\mathrm{T}$ é o número de períodos de produção). Uma das partições mais utilizadas é a partição por períodos. Estamos, portanto, traçando uma estratégia ótima para os primeiros períodos do horizonte de planejamento, considerando de forma relaxada as restrições de preparação dos períodos seguintes. Embora esta partição seja muito utilizada, dependendo dos dados do problema, devido a sua estrutura, ela pode resultar em soluções infactíveis, pois decisões de produção equivocadas no início do horizonte podem inviabilizar o atendimento da demanda em seus períodos finais. Na Figura 4.2 ilustramos esta situação. 


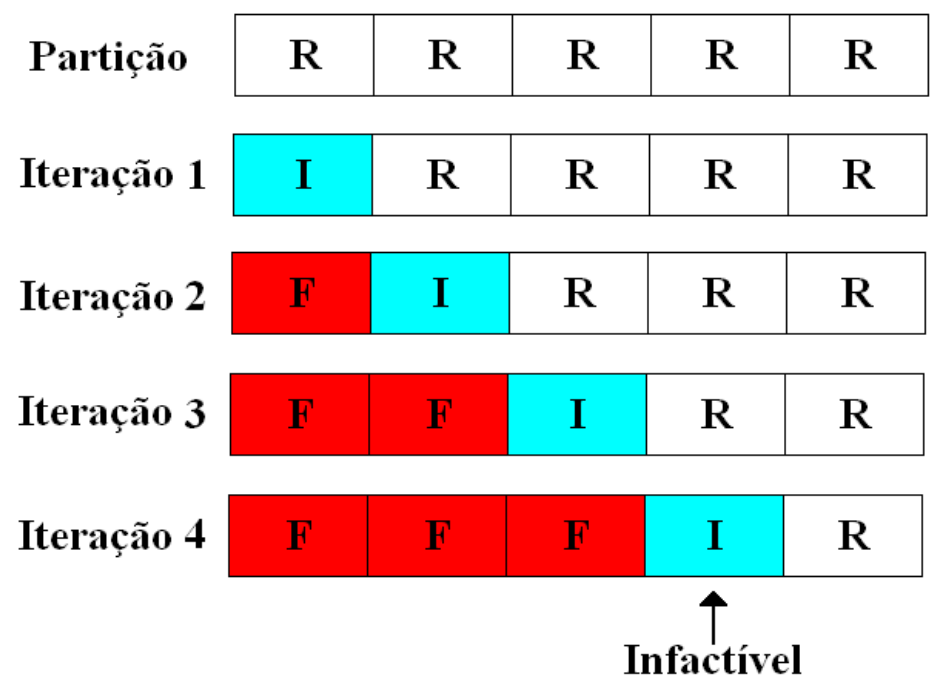

Figura 4.2 - Representação de infactibilidade no método relax-and-fix.

No entanto, se fosse permitido ao método que uma parte das variáveis inteiras de iterações anteriores pudesse ser alterada, teríamos, por um lado, um método mais flexível, mas por outro lado, devido ao número de variáveis inteiras que podem ser alteradas, um subproblema mais difícil de resolver.

Nossa proposta consiste em flexibilizar a fixação das variáveis de uma partição, adicionando a cada subproblema uma restrição do tipo local branching. Buscamos, além de evitar soluções infactíveis, encontrar soluções de melhor qualidade.

Primeiramente, não iremos mais fixar definitivamente as variáveis no método relax-and-fix, e para permitir que seja alterado no máximo um número fixo de variáveis inteiras, vamos utilizar uma restrição de local branching, adicionando a restrição (15) em cada iteração do método relax-and-fix. Neste caso, os conjuntos $Z^{0}$ e $Z^{1}$ são definidos com base no valor das variáveis das partições em que as variáveis já são inteiras.

A math-heurística proposta é resumida pelo Algoritmo 1, ilustrado na Figura 4.3. 


\begin{abstract}
Algoritmo 1: Relax-and-Fix+RLB
Passo 1: Criar uma partição para as variáveis binarias do problema.

Passo 2: Relaxar todas as variáveis binárias linearmente.

Passo 3: Inicializar os conjuntos $Z^{0}$ e $Z^{1}$ como vazios e $k=1$.

Passo 4: Selecionar a partição $k$ de variáveis relaxadas do problema e considerá-la como variáveis inteiras.

Passo 5: Se k>1 então adicionar a restrição (15) ao problema.

Passo 6: Resolver o subproblema inteiro misto. Se não for encontrada solução factível, retornar FALHA.

Passo 7: Atualizar os conjuntos $Z^{0}$ e $Z^{1}$ e $k=k+1$.

Passo 8: Caso ainda exista uma partição de variáveis relaxadas linearmente, retorne ao Passo 4, caso contrário, retornar a solução atual.
\end{abstract}

Figura 4.3: Descrição do Algoritmo 1.

A Figura 4.3 descreve de maneira geral o algoritmo proposto. Uma vez criada a partição das variáveis binárias do problema, elas são todas relaxadas linearmente antes da aplicação da primeira iteração do método. Na primeira iteração, os conjuntos $Z^{0}$ e $Z^{1}$ são vazios. Em cada uma das iterações do método, um novo grupo de variáveis inicialmente relaxadas linearmente torna-se inteiro. A restrição (15), atualizada e adicionada a cada iteração, permite que algumas destas variáveis mudem de valor, logo o método tem maior possibilidade de encontrar soluções factíveis (ou ainda soluções factíveis de melhor qualidade) para o problema principal, mas mantendo esta busca por soluções rápida, já que a busca é feita em uma região limitada.

Experimentalmente, notamos que as mudanças das variáveis inteiras, quando ocorrem, costumam ocorrer nos períodos imediatamente anteriores ao último período que deixou de ser relaxado e passou a ser inteiro.

Baseados nesse fato, também estamos propondo uma variante do método, que diminui o tempo de execução de cada subproblema, mantendo a qualidade das soluções obtidas. Como as mudanças nas variáveis inteiras costumam ocorrer nas partições imediatamente anteriores a partição atual, ao fixarmos as variáveis inteiras de partições mais distantes, estamos diminuindo a região de busca dos últimos subproblemas (onde a 
região de busca é maior), tornando a solução de cada subproblema mais rápida. A Figura 4.4 ilustra esquematicamente a ideia dessa heurística modificada

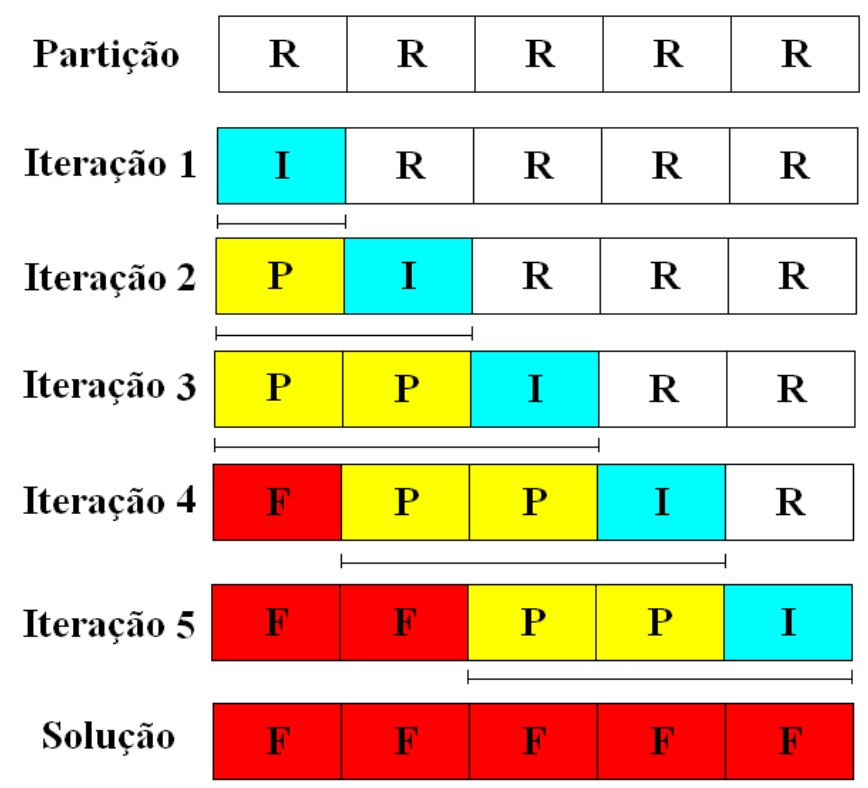

Figura 4.4: Representação esquemática da heurística modificada.

Na Figura 4.4, os retângulos brancos com a letra $\mathbf{R}$ em destaque representam variáveis relaxadas linearmente. Os retângulos azuis com a letra I em destaque representam variáveis inteiras. Os retângulos amarelos com a letra $\mathbf{P}$ em destaque representam variáveis inteiras que podem ter seus valores alterados na iteração corrente. Finalmente, os retângulos vermelhos com a letra $\mathbf{F}$ em destaque representam as variáveis que foram fixadas. As linhas abaixo de cada iteração representam quais variáveis inteiras estão sujeitas a mudanças na iteração corrente.

Para esta variante proposta, as variáveis inteiras são permanentemente fixadas $\beta$ iterações após terem sido fixadas pela primeira vez. Na Figura 4.5, estamos ilustrando uma situação onde $\beta=3$. É interessante notar que se $\beta=1$, estamos utilizando o método relax-and-fix puro. Este parâmetro $\beta$ é muito importante para a eficiência do método, uma vez que valores muito grandes de $\beta$ tornam a região de busca muito grande, e consequentemente, cada subproblema mais difícil de resolver, embora possam resultar em soluções de melhor qualidade. Ao passo que um valor muito pequeno de $\beta$ torna cada subproblema menor e mais fácil de resolver, mas, em geral, retorna soluções de qualidade inferior, além disso, quanto maior o valor de $\beta$, maior a chance de evitarmos 
infactibilidades. Assim sendo, é possível descrever o algoritmo da heurística modificada como:

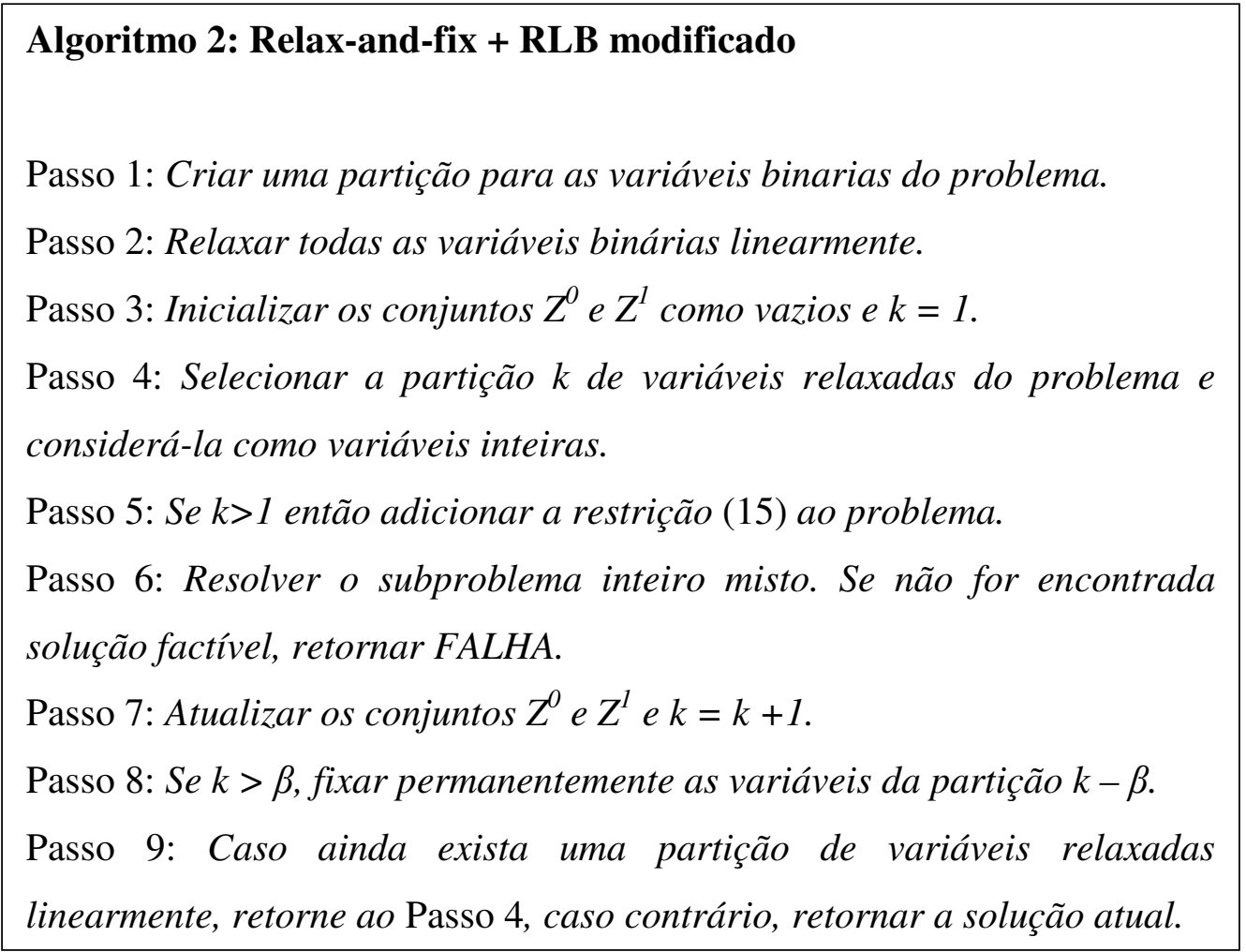

Figura 4.5: Descrição do Algoritmo 2.

Foram feitos experimentos computacionais preliminares para determinar quantas variáveis inteiras devemos incluir em cada partição. Pelos resultados obtidos, decidimos tomar grupos com cinco períodos de produção (ou seja, 5nm variáveis inteiras) para cada iteração do método relax-and-fix.

\subsection{Resultados Computacionais}

Com a finalidade de avaliar a qualidade da heurística proposta, implementamos a heurística em linguagem de modelagem e foram resolvidas as instâncias de (Muller, et al., 2012), as mesmas instâncias as quais a remodelagem teve dificuldades para resolver, utilizando-se o software de otimização CPLEX versão 12.4, em uma máquina com configurações Ubuntu 10.04 (64 bits), Intel Core i5 - 2300, 2.8GHz, 2Gb de RAM, com tempo limite máximo total de uma hora de execução. 
Em testes preliminares definimos uma partição tomando cinco períodos consecutivos, e com o valor de $\beta=3$, e tempo limite total de uma hora de execução. Assim, o tempo de execução de cada subproblema é dado em segundos, por $\frac{3600}{\left(\frac{T}{2}\right)}=\frac{18000}{T}$, onde T indica o número de períodos da instância testada.

A heurística relax-and-fix pura (sem restrições de local branching) foi testada para esta classe de instâncias, e retornou solução factível em apenas uma das 300 instâncias avaliadas. Logo, apresentamos apenas os resultados para a math-heurística proposta.

Estes resultados obtidos foram comparados com o melhor resultado obtido pelo CPLEX com o tempo limite de uma hora.

Primeiramente foram realizados testes considerando o problema sem a possibilidade de atrasos na entrega (backlogging). Os resultados obtidos estão apresentados na Tabela 4.1. A primeira, segunda e terceira colunas indicam respectivamente o número de plantas, de produtos e de períodos considerados em cada instância. A quarta e quinta colunas representam o GAP médio obtido pelo software CPLEX, com tempo limite de uma hora, e o número de instâncias nas quais ao menos uma solução inteira factível é obtida. Finalmente, nas últimas duas colunas estão reportados o desvio médio percentual em relação às soluções inteiras obtidas, e o número de instâncias para as quais a math-heurística obteve solução factível.

O desvio médio percentual é dado pela expressão:

$$
\text { Desvio }=\frac{\sum_{i=1}^{k} \frac{\left(Z_{P_{i}}-Z_{H_{i}}\right) * 100}{Z_{P_{i}}}}{k}
$$

onde:

k: indica o número de instâncias para as quais os dois métodos encontraram solução factível dentro do tempo limite de uma hora;

$Z_{P_{i}}$ : indica o valor da i-ésima solução factível obtida pelo CPLEX;

$\bar{Z}_{E_{i}}$ : indica o valor da i-ésima solução obtida pela math-heurística proposta.

O primeiro resultado obtido é que a math-heurística obtém uma solução factível para o problema para todas as 300 instâncias testadas, ao passo que o software CPLEX 
obtém solução factível em 267 das instâncias. Além disso, as soluções obtidas são de boa qualidade, quando comparadas às soluções obtidas pelo CPLEX. Em grande parte dos testes, a math-heurística encontra uma solução de melhor qualidade, sendo em média aproximadamente $0,48 \%$ melhor. 
Tabela 4.1 - Resultados obtidos para problemas sem possibilidade de atraso na entrega: Instâncias de (Muller, , 2012).

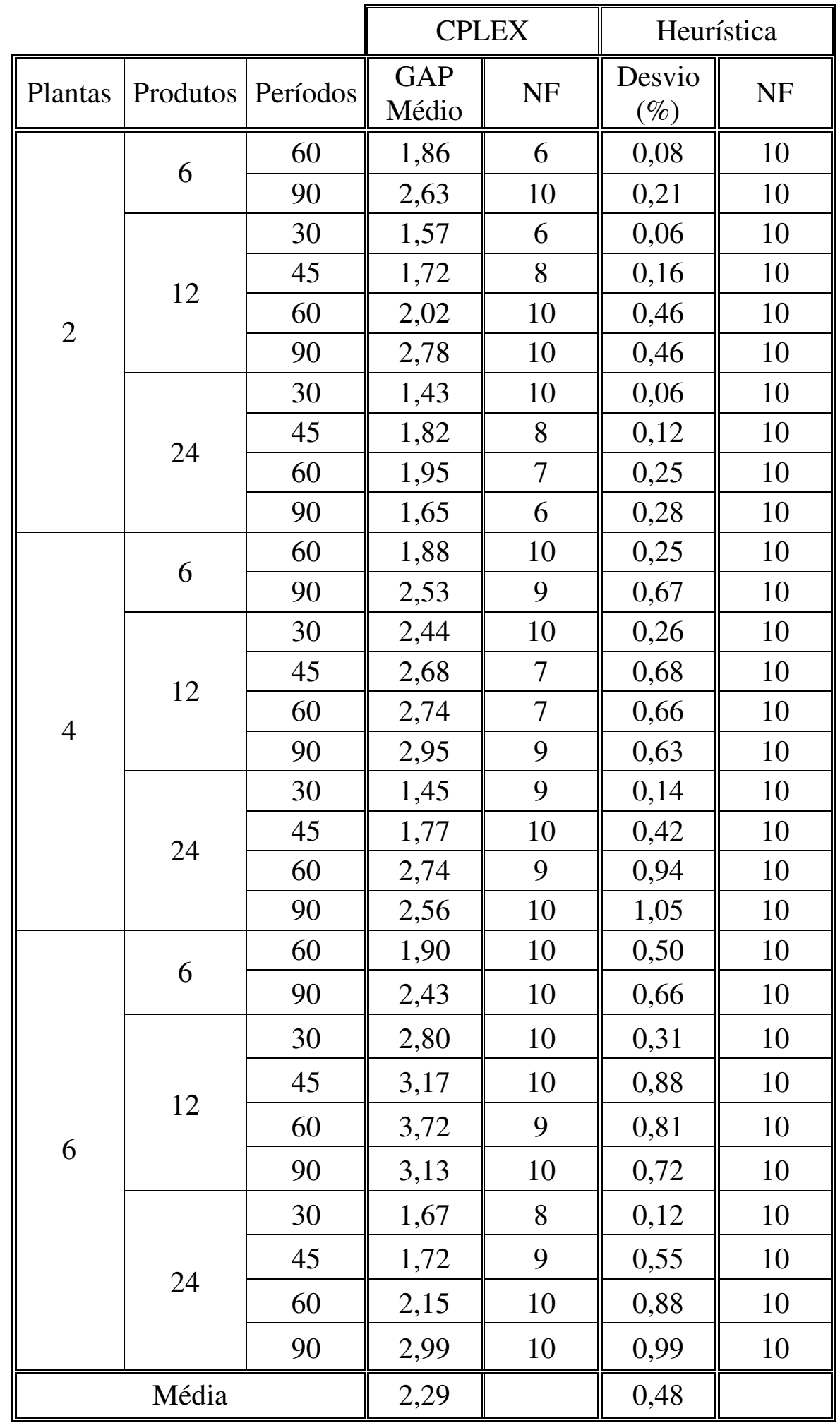


Também foram avaliadas as mesmas instâncias, considerando a possibilidade de atrasos na entrega (backlogging). A Tabela 4.2 mostra os resultados obtidos para esses testes, mantendo as informações conforme indicadas na Tabela 4.1.

Tabela 4.2 - Resultados obtidos para problemas com possibilidade de atraso na entrega: Instâncias de (Muller, et al., 2012).

\begin{tabular}{|c|c|c|c|c|c|c|}
\hline \multirow[b]{2}{*}{ Plantas } & \multirow[b]{2}{*}{ Produtos } & \multirow[b]{2}{*}{ Períodos } & \multicolumn{2}{|c|}{ CPLEX } & \multicolumn{2}{|c|}{ Heurística } \\
\hline & & & GAP & NF & $\begin{array}{c}\text { Desvio } \\
(\%)\end{array}$ & NF \\
\hline \multirow{10}{*}{2} & \multirow{2}{*}{6} & 60 & 1,22 & 10 & $-2,31$ & 10 \\
\hline & & 90 & 1,69 & 9 & $-2,14$ & 10 \\
\hline & \multirow{4}{*}{12} & 30 & 1,28 & 10 & $-3,30$ & 9 \\
\hline & & 45 & 1,64 & 10 & $-2,74$ & 9 \\
\hline & & 60 & 1,96 & 10 & $-2,35$ & 10 \\
\hline & & 90 & 2,15 & 10 & $-2,05$ & 10 \\
\hline & \multirow{4}{*}{24} & 30 & 1,38 & 10 & $-3,08$ & 10 \\
\hline & & 45 & 1,40 & 10 & $-2,40$ & 10 \\
\hline & & 60 & 1,67 & 8 & $-1,99$ & 10 \\
\hline & & 90 & 1,29 & 8 & $-1,54$ & 10 \\
\hline \multirow{10}{*}{4} & \multirow{2}{*}{6} & 60 & 1,24 & 10 & $-0,74$ & 10 \\
\hline & & 90 & 1,79 & 9 & $-0,67$ & 10 \\
\hline & \multirow{4}{*}{12} & 30 & 1,37 & 10 & $-0,72$ & 10 \\
\hline & & 45 & 1,76 & 9 & $-0,66$ & 10 \\
\hline & & 60 & 1,98 & 10 & $-1,64$ & 10 \\
\hline & & 90 & 2,21 & 10 & $-1,48$ & 10 \\
\hline & \multirow{4}{*}{24} & 30 & 1,45 & 9 & $-1,34$ & 10 \\
\hline & & 45 & 1,46 & 10 & $-0,69$ & 10 \\
\hline & & 60 & 1,80 & 10 & $-0,52$ & 10 \\
\hline & & 90 & 1,80 & 10 & $-0,40$ & 10 \\
\hline \multirow{10}{*}{6} & \multirow{2}{*}{6} & 60 & 1,25 & 10 & $-0,36$ & 10 \\
\hline & & 90 & 1,90 & 9 & $-0,67$ & 10 \\
\hline & \multirow{4}{*}{12} & 30 & 1,47 & 10 & $-0,59$ & 10 \\
\hline & & 45 & 1,88 & 10 & $-0,51$ & 10 \\
\hline & & 60 & 1,99 & 10 & $-1,34$ & 10 \\
\hline & & 90 & 2,29 & 10 & $-1,30$ & 10 \\
\hline & \multirow{4}{*}{24} & 30 & 1,52 & 8 & $-1,62$ & 10 \\
\hline & & 45 & 1,53 & 10 & $-0,53$ & 10 \\
\hline & & 60 & 1,93 & 10 & $-0,56$ & 10 \\
\hline & & 90 & 2,52 & 10 & $-0,43$ & 10 \\
\hline \multicolumn{3}{|c|}{ Média } & 1,69 & & $-1,36$ & \\
\hline
\end{tabular}


Para essa classe de testes, a math-heurística conseguiu encontrar soluções factíveis para 298 instâncias das 300 testadas, ou seja 99,33\% das instâncias testadas, ao passo que o CPLEX encontrou soluções factíveis para 289 instâncias.

No entanto, destacamos que o desempenho da math-heurística não se mostrou tão bom para esse caso quanto havia se mostrado para o problema sem possibilidade de atrasos na entrega. A math-heurística não determinou soluções de melhor qualidade para nenhuma das instâncias em que o CPLEX encontrou solução factível. No entanto, as soluções encontradas são de boa qualidade, estando em média auma diferença de 1,36\% aproximadamente.

Além disso, nota-se que os problemas de maior magnitude apresentam resultados melhores em relação aos problemas de menor dimensão, como ocorria no problema sem backlogging.

Essa diferença entre os resultados obtidos para os problemas com e sem possibilidade de atrasos na entrega pode ser explicada devido a natureza dos problemas com e sem possiblidade de atraso. Ao considerarmos o problema sem possibilidade de atrasos, estamos trabalhando com um conjunto factível reduzido em relação ao problema com possibilidade de atrasos na entrega. Assim sendo, em cada partição, fica mais fácil determinar a solução ótima do subproblema (ou ao menos uma factível de melhor qualidade) dentro do tempo limite de cada iteração, e portanto, a heurística apresenta soluções de melhor qualidade para o problema sem a possiblidade de atrasos na entrega, quando comparada com o mesmo problema, com a possibilidade de atrasos.

\subsection{Conclusões}

Apresentamos neste capítulo uma math-heurística baseada nos métodos relaxand-fix e em local branching como proposta para a resolução do problema de dimensionamento de lotes para o problema de múltiplas plantas, capacitado e com tempo de preparação de máquina.

Testes computacionais realizados mostraram que o método proposto consegue obter soluções factíveis de boa qualidade para as instâncias avaliadas, obtendo soluções factíveis com maior frequência que o software CPLEX, sendo que, para os casos onde é considerada a possibilidade de atrasos na entrega, a math-heurística consegue 
determinar soluções de melhor qualidade que o software, quando ambas são confrontadas sob as mesmas condições computacionais.

Finalmente, destacamos que a heurística proposta obteve melhor desempenho nos problemas de maior dimensão, e também teve um desempenho melhor para os problemas em que o atraso no atendimento da demanda não é permitido. 


\section{Conclusões e Pesquisas Futuras}

Esta dissertação aborda o problema de dimensionamento de lotes com múltiplas plantas, múltiplos itens e múltiplos períodos. O ambiente de produção apresenta restrições de capacidade, e a fabricação dos produtos depende da preparação prévia para a produção, o que incorre em custos e tempos de preparação. O objetivo é minimizar a soma dos custos de produção, estoque, preparação e transporte de produtos em todas as plantas, de modo a obter um planejamento de produção mais eficiente em relação ao planejamento individual de cada planta.

Apesar do potencial de aplicações práticas, este problema foi pouco abordado na literatura, especialmente devido a sua alta complexidade, que torna o problema de difícil solução. Neste trabalho foram apresentadas duas contrubuições para a resolução deste problema, a primeira é a remodelagem do problema através da idéia de localização de facilidades. E a segunda, é um método de solução para o problema.

O modelo proposto em (Sambasivan \& Yahya, 2005) foi reformulado de acordo com a proposta de (Krarup \& Bilde, 1977). Testes computacionais com três diferentes grupos de instâncias, mostraram que o modelo tem relaxações lineares de melhor qualidade. Além disso, destacamos que com a remodelagem do problema, é possível resolver na otimalidade as instâncias de menor dimensão, utilizando-se o software CPLEX versão 12.4.

Para tratar as instâncias de maior dimensão, foi proposta uma math-heurística, baseada no método relax-and-fix e na restrição de local branching. Este método foi avaliado para o grupo de instâncias de maior dimensão, e encontrou um número maior de soluções factíveis quando comparado à solução do problema utilizando o software CPLEX versão 12.4 .

Em relação ao método proposto, uma possível linha de investigação é adaptá-lo para outros problemas de dimensionamento de lotes, e buscar uma aplicação real para aplicação da math-heurística proposta, preferencialmente em um problema de dimensões grandes, uma vez que nos testes realizados o método se apresentou melhor em instâncias de maiores dimensões.

Além disso, uma investigação adicional pode ser realizada em relação à própria construção do método, em relação ao momento e à quantidade de variáveis fixadas. A escolha, feita neste trabalho sempre a priori, pode ser feita no decorrer do método, em 
função de seu desenvolvimento tornando-o adaptativo. Uma investigação sobre a eficiência destas mudanças pode torná-lo ainda mais eficiente. 


\section{Referências Bibliográficas}

Aggarwal, A. \& Park, J. K., 1993. Improved algorithms for economic lot size problems. Operations Research, 41, pp. 549-571.

Araujo, S. A., Arenales, M. N. \& Clark, A. R., 2008. Lot-sizing and furnace scheduling in small foundries. Computers \& Operations Research, 35, pp. 916 - 932.

Armentano, V. A., França, P. M. \& Toledo, F. M. B., 1999. A network flow model for the capacitated lot-sizing problem. International Journal of Management Science, 27, pp. 275-284.

Bertaldi, P., Ghiani, G., Grieco, A. \& Guerreiro, E., 2008. Rolling-horizon and fix-andrelax heuristics for the parallel machine lot-sizing and scheduling problem with sequence-dependet setup costs. Computers and Operations Research, 35, pp. 3644 3656.

Brahimi, N., Dauzere-Perez, S., Najid, N. M. \& Nordli, A., 2006. Single item lot sizing problems. European Journal of Operational Research, 168, pp. 1 - 16.

Buschkühl, L., Sahling, F., Helber, S. \& Tempelmeier, H., 2010. Dynamic capacitated lot-sizing problems: a classification and review of solution approaches. Operational Research 32, pp. $231-261$.

Camargo, V. C. B., Mattiolli, L. \& Toledo, F. M. B., 2012. A knapsack problem as a tool to solve the production planning problem in small foundries. Computers \& Operations Research, 39, pp. 86 - 92.

Carreno, J. J., 1990. Economic lot scheduling for multiple products on paralell identical processors. Management Science, 36, pp. 348-358.

Diaby, M., Bahl, H. C., Karwan, M. H. \& Zionts, S., 1992a. A Lagrangean Relaxation Approach for Very-Large-Scale Capacitated Lot-Sizing. Management Science, 38, pp. 1329-1340.

Diaby, M., Bahl, H. C., Karwan, M. H. \& Zionts, S., 1992b. Capacitated lot-sizing and scheduling by Lagrangean Relaxation. European Journal of Operational Research, 59, pp. 444-458.

Dillenberger, C., Escudero, L. F., Zhang, W. \& Wollensak, A., 1994. On pratical resource allocation for production planning and scheduling with period overlapping setups. European Journal of Operational Research, 75, pp. 275-286.

Drexl, A. \& Kimms, A., 1997. Lot sizing and scheduling - survey and extensions. Journal of Operational Research, 99, pp. 221 - 235. 
Evans, J. R., 1985b. Network-based optimization algorithms for the capacitated multiitem lot sizing problem. Computers \& Industrial Engeneering, 9, pp. 297-305.

Federgruen, A. \& Tzur, M., 1991. A simple foward algorithm to solve general dynamic lot sizing models with $\mathrm{n}$ periods in $\mathrm{O}(\mathrm{n} \log (\mathrm{n}))$ or $\mathrm{O}(\mathrm{n})$ time. Management Science, 37, pp. 909-925.

Ferreira, D., Morabito, R. \& Rangel, S., 2009. Solution approaches for the soft drink integrated production lot sizing and scheduling problem. European Journal of Operational Research, 196, pp. 697 - 706.

Fischetti, M. \& Lodi, A., 2003. Local Branching. Mathematical. Programming, 98, pp. 23-47.

Hindi, K. S., 1995. Computationally Efficient Sollution of the Multi-Item Capacitated Lot-Sizing Problem. Computers \& Industrial Engeneering, 28, pp. 709-719.

Jans, R. \& Degraeve, Z., 2008. Modeling industrial lot sizing problems: a review. International Journal of Production Research, 46, March, pp. 1619 - 1643.

Karimi, B., Ghiomi, S. M. T. F. \& Wilson, J. M., 2003. The capacitated lot sizing problem: a review of models and algorithms. Omega, 31, pp. $365-378$.

Krarup, J. \& Bilde, O., 1977. Sharp lower bounds and efficient algorithms for the simple plant location problem. Annals of Discrete Mathematics, 1, pp. 79 - 97.

Lozano, S., Larraneta, J. \& Onieva, L., 1991. Primal-dual approach to the single-level capacitated lot-sizing problem. European Journal of Operational Research, 51, pp. 354366.

Maes, J., McCain, J. O. \& Wassenhove, L. N. V., 1991. Multilevel capacitated lotsizing complexity and LP-based heuristics. European Journal of Operacional Research, 53, pp. 131-148.

Mohammadi, M., Fatemi Ghiomi, S. M. T., Karimi, B. \& Torabi, S. A., 2010. Rollinghorizon and fix-and-relax heuristics for the multi-product, multi-level capacitated lotsizing problem with sequence-dependent setups. Journal of Intelligent Manufacturing, 21, pp. 501-510.

Muller, L. F., Spoorendonk, S. \& Pisinger, D., 2012. A hybrid adaptative large neighborhood search heuristic for lot-sizing with setup times. European Journal of Operational Research 218, pp. 614-623.

Nascimento, M. C. V., Resende, M. G. C. \& Toledo, F. M. B., 2010. GRASP heuristic with path-relinking for the multi-plant capacitated lot sizing problem. European Journal of Operacional Research, 200, pp. 747 - 754.

Özdamar, L. \& Birbil, S. I., 1998. Hybrid heuristics for the capacitated lot sizing and loading. European Journal od Operacional Research, 110, pp. 525 - 547. 
Poltroniere, S. C., Poldi, K. C., Toledo, F. M. B. \& Arenales, M. N., 2008. Coupling cutting stock and lot sizing problems in the paper industry. Annals of Operations Research 157, pp. 91 - 104.

Sambasivam, M. \& Schimidt, C. P., 2002. A heuristic procedure for solving multi-plant, multi-item, multi-period, capacitated, lot-sizing problems. Asia - Pacific Journal of Operacional Research, 19, pp. 87 - 105.

Sambasivan, M. \& Schmidt, C. P., 1999. A solution procedure to solve uncapacitated lot sizing for multi-plant, multi-period problems with inter-plant transfers, University of Putra Malaysia: Faculty of Economics.

Sambasivan, M. \& Yahya, S., 2005. A Lagrangean-based heuristic for multi-plant, multi-item, multi-period capacitated lot-sizing problems with inter-plant transfers. Computers \& Operations Research, 32, pp. 537-555.

Santos-Meza, E., Santos, M. O. \& Arenales, M. N., 2002. A lot-sizing problem in an automated foundry. European Journal of Operational Research, 139, pp. 490 - 500.

Souza, K. X. S. \& Armentano, V. A., 1994. Multi-item capacitated lot-sizing by a Cross decomposition based algorithm. Annals of Operations Research, 50, pp. 557-574.

Sung, C. S., 1986. A single-product parallel-facilities production-planing model. International Journal of Systems Science, 17, pp. 983-989.

Toledo, F. M. B. \& Armentano, V. A., 2006. A Lagrangean-based heuristic for the capacitated lot-sizing problem in parallel machines.. European Journal of Operacional Research, 175, pp. 1070 - 1083.

Toledo, F. M. B., Santos, M. O., Arenales, M. N. \& Seleghim, P., 2008. Logística de distribuição de água em redes urbanas - Racionalização energética. Pesquisa Operacional, 28, pp. 75 - 91.

Toso, E. A., Morabito, R. \& Clark, A., 2009. Lot-sizing and sequencing optimization at an animal-feed plant. Computers \& Industrial Engineering, 57, pp. 813 - 821.

Trigeiro, W. W., 1987. The effect of setup time on production lot sizes. Production and Inventory Management, 28, pp. 50 - 52.

Trigeiro, W. W., Thomas, L. J. \& MacClain, J. O., 1989. Capacitated lot sizing with setup times. Management Science, 35, pp. 353-366.

Wagelmans, A., Van Hoesel, S. \& Kolen, A., 1992. Economic Lot Sizing: An O(n $\log (\mathrm{n}))$ algorithm that runs in linear time in the Wagner-Within case. Operations Research, 40, pp. 145-156.

Wagner, H. M. \& Within, T. M., 1958. Dynamic Version of the Economic Lot Size Model. Management Science, 5, pp. 89-96. 
Wosley, L. A., 1995. Progress with single-item lot-sizing. European Journal of Operational Research, 86, pp. 395-401.

Wu, T. \& Shi, L., 2011. Mathematical models for capacitated multi-level production planning problems with linked lot-sizing. International Journal of Production Research, 49 , pp. 6227 - 6247.

Zangwill, W. I., 1966. A Deterministic Multi-Period Production Scheduling Model with Backlogging. Management Science, 13, pp. 105-119.

Zangwill, W. I., 1969. A backlogging model and a multi-echelon model of a dynamic economic lot size production system - a network approach. Management Science, 15, pp. 506-527. 